\title{
Comparison of environmental and isolate Sulfobacillus genomes reveals diverse carbon, sulfur, nitrogen, and hydrogen metabolisms
}

\author{
Nicholas B Justice ${ }^{1,2^{*}}$, Anders Norman ${ }^{1,3}$, Christopher T Brown ${ }^{1}$, Andrea Singh ${ }^{1}$, Brian C Thomas ${ }^{1}$ and Jillian F Banfield
}

\begin{abstract}
Background: Bacteria of the genus Sulfobacillus are found worldwide as members of microbial communities that accelerate sulfide mineral dissolution in acid mine drainage environments (AMD), acid-rock drainage environments (ARD), as well as in industrial bioleaching operations. Despite their frequent identification in these environments, their role in biogeochemical cycling is poorly understood.

Results: Here we report draft genomes of five species of the Sulfobacillus genus (AMDSBA1-5) reconstructed by cultivation-independent sequencing of biofilms sampled from the Richmond Mine (Iron Mountain, CA). Three of these species (AMDSBA2, AMDSBA3, and AMDSBA4) have no cultured representatives while AMDSBA1 is a strain of S. benefaciens, and AMDSBA5 a strain of $S$. thermosulfidooxidans. We analyzed the diversity of energy conservation and central carbon metabolisms for these genomes and previously published Sulfobacillus genomes. Pathways of sulfur oxidation vary considerably across the genus, including the number and type of subunits of putative heterodisulfide reductase complexes likely involved in sulfur oxidation. The number and type of nickel-iron hydrogenase proteins varied across the genus, as does the presence of different central carbon pathways. Only the AMDSBA3 genome encodes a dissimilatory nitrate reducatase and only the AMDSBA5 and $\mathrm{S}$. thermosulfidooxidans genomes encode assimilatory nitrate reductases. Within the genus, AMDSBA4 is unusual in that its electron transport chain includes a cytochrome bc type complex, a unique cytochrome coxidase, and two distinct succinate dehydrogenase complexes.
\end{abstract}

Conclusions: Overall, the results significantly expand our understanding of carbon, sulfur, nitrogen, and hydrogen metabolism within the Sulfobacillus genus.

\section{Background}

Species of the Sulfobacillus genus are Gram-positive spore forming bacteria belonging to the order Clostridiales, and are found globally in acidic environments such as thermal springs [1], hydrothermal vents [2], solfatara fields [3], and acid mine drainage (AMD) environments [4]. Sulfobacillus species are also frequently found in industrial reactors used in bioleaching operations [5]. As such, an understanding of their physiology is of both environmental and biotechnological importance.

In the AMD environment of the Richmond Mine (Iron Mountain, CA) Sulfobacillus are members of acidophilic,

\footnotetext{
* Correspondence: njustice@berkeley.edu

${ }^{1}$ Department of Earth and Planetary Science, University of California, Berkeley, CA 94720, USA

${ }^{2}$ Present Address: Physical Biosciences Division, Lawrence Berkeley National Lab, Berkeley, CA, USA

Full list of author information is available at the end of the article
}

metal-tolerant microbial consortia that promote sulfidemineral dissolution. In contrast to the dominant ironoxidizing Leptospirillum bacteria, or certain members of the heterotrophic Thermoplasmatales archaea, Sulfobacillus generally appear in much lower relative abundances, having been identified only through 16S rRNA clone libraries, fluorescent in situ hybridization [6-8], and as unresolved fragments assembled from metagenomic sequence information [9]. As their namesake implies, they are likely key players in sulfur cycling, yet relatively little is known about their metabolic pathways or how these vary across the Sulfobacillus genus.

Key features of Sulfobacillus metabolism have been described for several isolated Sulfobacillus species-S. sibiricus [10], S. thermotolerans [11], S. acidophilus [1], S. thermosulfidooxidans [12], and S. benefaciens [13]. Each is a facultative anaerobe capable of assimilation of 
organic and inorganic forms of carbon, deriving energy from aerobic oxidation of iron and various sulfur species (e.g., tetrathionate and elemental sulfur), as well as from ferric iron respiration $[10,11,14,15]$, and possibly fermentation [15]. Other phenotypic differences such as metal tolerance, temperature tolerance, and use of different carbon compounds set these organisms apart $[5,11,13]$. Genomes for two of these species-S. acidophilus and S. thermosulfidooxidans-have been previously reported [2,16-18] and an additional S. thermosulfidooxidans genome is deposited in the publicly available "Integrated Microbial Genomes and Metagenomes" (IMG) database [19]. Here we present five new draft genomes of Sulfobacillus organisms assembled from metagenomic data. We analyze these genomes in the context of the previously published genomes of Sulfobacillus isolates, and describe key elements of carbon, sulfur, nitrogen, and hydrogen metabolism. In addition to illuminating the metabolic potential of a strain of S. benefaciens, and three other novel Sulfobacillus spp., this work provides one of the first genome-inferred reconstructions of Gram-positive, acidophilic sulfur oxidation pathways, and highlights both conserved and divergent metabolisms found in organisms of the Sulfobacillus genus.

\section{Methods}

DNA was collected from the 5 way site at Iron Mountain Mine [20] and extracted as previously described [21]. This sample is referred to as ' 5 way fungal streamer', and comprised the majority of sequencing information used in the genome assembly. Briefly, $4-5 \mathrm{ml}$ of frozen biofilm was washed and thawed with cold acidified 0.9\% $\mathrm{NaCl}\left(\mathrm{pH} 1.0, \mathrm{H}_{2} \mathrm{SO}_{4}\right)$, homogenized with a $16 \mathrm{G}$ needle in cold $\mathrm{pH} 7.0$ phosphate buffered saline, and finally ground in a mortar and pestle in liquid nitrogen. The powdered biofilm pellets were then extracted with a PowerSoil DNA isolation kit (MoBio Laboratories, Carlsbad, CA, USA). Illumina library preparation sequencing was carried out according to JGI protocols [21,22].

Three flow cells of Illumina HiSeq were used to obtain $\sim 90$ million paired-end 76 bp reads $(8.46 \mathrm{~Gb})$. An additional overlapping paired-end library was sequenced to generate longer sequence reads. The initial paired-end reads were merged using SeqPrep [23] into $\sim 32$ million long reads $(6.17 \mathrm{~Gb})$ with a median length of 194 bp. Reads from 17 additional previously published metagenomic datasets were used to assist binning and assembly [24].

\section{Metagenomic sequence assembly}

A total of 854 million paired-end (PE) Illumina reads (98 Gb) pooled from the 18 samples described above were assembled de novo using the Iterative De-Bruijn
Assembler optimized for single-cell and metagenomic assemblies (idba-ud) [25]. A kmer-range of 19-99 was used with the pre-correction option enabled. All PE-reads were quality-trimmed using Sickle (sickle pe options -q 15 and -l 40) prior to assembly. PE-reads were mapped back to the resulting scaffolds using bwa [26]. All scaffolds larger than $1500 \mathrm{bp}$ were binned using emergent self-organizing maps (ESOM) trained on tetranucleotide frequencies as previously described [9]. Briefly, larger sequences were subdivided into $10 \mathrm{~kb}$ fragments, and trained for 100 epochs using the k-batch training method, along with any leftover fragments exceeding $3 \mathrm{~kb}$ in length. To minimize noise, sequences in the range 1500-3000 bp were not included during training, but instead projected onto the trained weight vectors generated with the larger fragments. In order to separate individual Sulfobacillus bins, a discrete bin containing putative members of the order Clostridiales, was isolated and further binned using log-normalized sample abundance patterns from 18 different samples, this time including fragments down to $1500 \mathrm{bp}$. Five distinct sub-bins (AMDSBA1, AMDSBA2, AMDSBA3, AMDSBA4 and AMDSBA5) were individually reassembled de novo using every read-pair that mapped within these respective bins. Additionally, because of its high similarity to S. thermosulfidooxidans DSM9293, all PE-reads mapping to this organism were included in the AMDSBA5 sub-assembly as well. Due to its considerably lower abundance, AMDSBA2 was sub-assembled using PE-reads as described above, but with the inclusion of additional reads from the overlapping HiSeq PE library that mapped within AMDSBA2 bin (median read length $194 \mathrm{bp}$ ). Scaffolds belonging to the Clostridiales bin, but not to the 5 putative genome bins were not reassembled. In order to identify areas of inconsistent coverage possibly indicative of chimeric assemblies, reads were mapped back to reassembled genomes and scaffolds binned a second time again using time-series abundance data. The resulting ESOM map was used to identify scaffold fragments that binned inconsistently, suggestive of chimeric assemblies. These scaffolds were manually checked with stringent paired read mapping (100\% identity) to either confirm the assembly or identify points where chimeras might occur.

\section{Metabolic analysis}

Open reading frames on assembled contigs for Iron Mountain Sulfobacillus and S. thermosulfidooxidans strain 'Cutipay' were predicted using Prodigal [27], and tRNAs predicted with tRNAscan [28]. Proteins were then compared with BLAST [29] to UniRef90 [30] and KEGG [31] with matches greater than 60 bits being reported. Reverse BLASTs were also used to identify reciprocal best BLAST hits. Proteins were further analyzed with InterProScan 
[32] to identify conserved domains. Protein sequences and corresponding annotations for S. thermosulfidooxidans AT-1 (DSM9293), S. acidophilus TPY, and S. acidophilus NAL (DSM10332) were downloaded from the Integrated Microbial Genomes database available at http://img.jgi. doe.gov/ [33]. While S. thermosulfidooxidans strain 'Cutipay' and S. acidophilus TPY were included in most analyses here, results focus on the high quality genomes of the type strains S. thermosulfidooxidans AT-I (DSM9293) and $S$. acidophilus NAL (DSM10332). Unless otherwise specified, all references to $S$. thermosulfidooxidans and $S$. acidophilus in the text refer specifically to these type strains.

Orthologs shared between species were identified with amino acid sequence searches between all pairwise combinations of genomes using USEARCH [34]. Reciprocal best hits between each pair of genomes were considered orthologs if the alignments of their sequences had an E-value less than 0.01, a Bit score greater than 40 , and covered at least $65 \%$ of each amino acid sequence.

\section{Protein phylogenetic analysis}

All protein trees were made by first aligning protein sequences with MUSCLE [35]. Next, alignments were trimmed using Gblocks [36,37], which stringently curates the alignment to phylogenetically informative sites. For each alignment, the optimum amino acid substitution model was estimated using ProtTest [38]. All trees were generated using RAxML [39] using the PROTCAT rate model and the ProtTest-determined amino acid substitution model. Support was evaluated using 500 bootstrap replications in RAxML. Trees were visualized in iTOL [40].

The concatenated ribosomal protein tree was made using 16 core ribosomal proteins (rpL2, 3, 4, 5, 6, 14, $15,16,18,22,24, \mathrm{rpS} 3,8,10,17,19)$, selected based on low frequencies of lateral gene transfer $[41,42]$. These proteins were aligned with MUSCLE [35], manually trimmed, then concatenated, resulting in an 2,052 residue alignment. Phylogenetic relationships were analyzed using RAxML using the $\mathrm{LG}+\alpha+\gamma$ substitution model, and nodal support determined with 500 bootstrap replicates. Trees were visualized in FigTree (available: http://tree.bio.ed.ac.uk/software/figtree/)

\section{S rRNA gene sequence analysis}

Near full-length 16S rRNA sequences for Sulfobacillus species were generated using EMIRGE-an iterative template-guided assembler that probabilistically generates $16 \mathrm{~S}$ rRNA gene sequences using a $16 \mathrm{~S}$ rRNA database [21]. First, potential 16S rRNA gene regions were found by a BLAST of all assembled contigs against SILVA db v. 108 [43]. Reads that mapped to these regions were extracted and trimmed with Sickle (available https://github.com/najoshi/sickle), allowing only paired-end reads with length $>60$ and quality scores $>20$. For the reference database, 186 sequences were downloaded from the SILVA SSU database representing the 174 sequences of 'Family XVII Incertae Sedis' (the family to which the Sulfobacillus genus belong) as well as twelve representative sequences of other organisms known to be represent the majority of the Richmond Mine microbial communities, including Leptospirillum species and Archaea of the ARMAN and Thermoplasmatales lineages. Potential chimeric sequences in this database were removed with DECIPHER [44] and UCHIME [45] [searched against the 2011 Greengenes database [46]]. EMIRGE was run in 50 iterations with a “join_threshold” parameter of 0.99 .

Using the QIIME software suite [47], the EMIRGEgenerated sequences and representative sequences of Sulfobacillus isolates were aligned using PyNAST with default parameters [48], and filtered with filter_alignment.py in QIIME. Edges were trimmed leaving 1,154 unambiguously aligned positions. A maximum likelihood tree was generated with RAxML using the GTRCAT model. Nodal support was evaluated with 500 bootstrap replicates. Trees were visualized in FigTree.

\section{Microscopy}

Environmental samples were fixed and stained with lineage-specific fluorescent in situ hybridization (FISH) probes to examine Sulfobacillus populations, as described previously [49]. Sulfobacillus (SUL230; 5' - TAA TGGGCCGCGRGCYCC) and archaeal-specific probes (ARC915; 5'-GTGCTCCCCCGCCAATTCCT) have been previously reported $[7,50]$.

\section{Results}

\section{Overall genome statistics and phylogeny}

We reconstructed the near complete genomes of five Sulfobacillus species from the Richmond Mine, represented as five discrete bins on abundance-pattern ESOMs (Figure 1). Each genome had between 129 and 409 scaffolds with coverage ranging from 10 to $258 \mathrm{X}$ (Table 1 ). Genomes were between 3.07 and $4.56 \mathrm{Mbp}$, and encoded between 3,312 and 4,629 protein-coding genes. Additional file 1: Figure S1 summarizes the completeness of each genome estimated using the presence of a set of conserved, single copy genes with low frequency of horizontal gene transfer $[41,42]$.

Based on phylogenetic trees of concatenated protein alignments and EMIRGE-generated 16S rRNA genes, AMDSBA5 was classified as a strain of $S$. thermosulfidooxidans (99.7\% 16S rRNA gene similarity), AMDSBA1 was classified as a strain of S. benefaciens (100\% $16 \mathrm{~S}$ rRNA gene similarity), and AMDSBA3 was shown to 


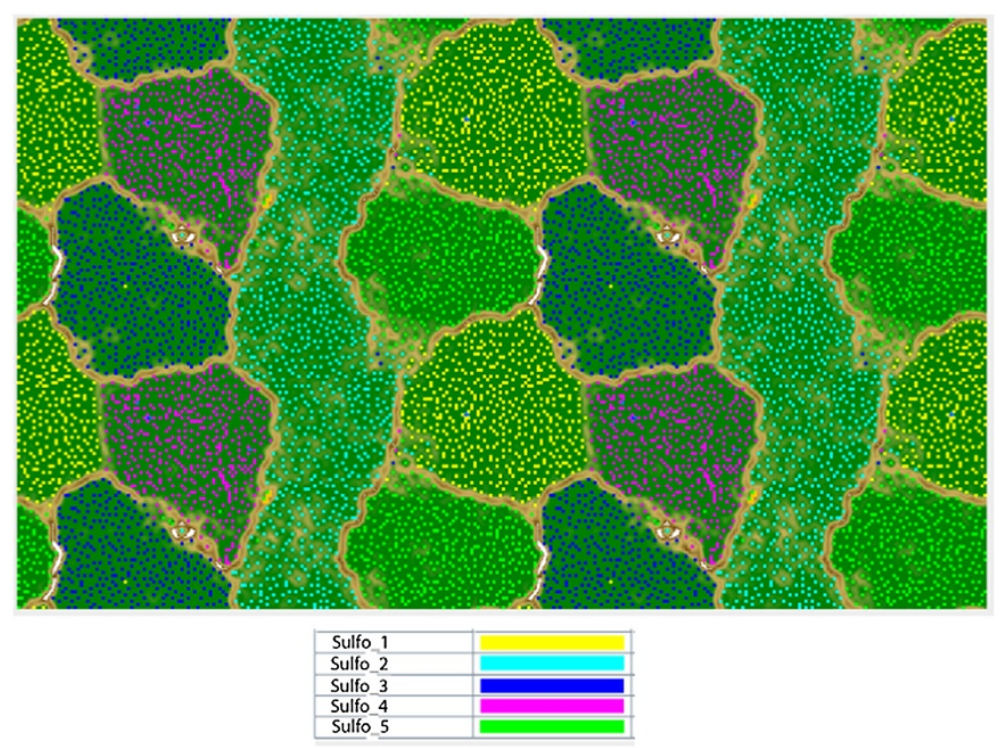

Figure 1 Emergent self-organizing map (ESOM) of Clostridiales sequence fragments binned by time-series abundance patterns (>1500 bp). Note that the map is continuous (top and bottom edges, left and right edges). Each point represents a sequence fragment with colors indicative of the genome bin it belongs too (see legend below).

related to be most closely related to $S$. acidophilus strains (97.2\% 16S rRNA gene similarity, Figure 2A and B, Additional file 2: Table S1). AMDSBA2 may represent another strain of $S$. thermosulfidooxidans with 99.2\% $16 \mathrm{~S}$ similarity, while AMDSBA4 is at least 97\% divergent from any Sulfobacillus isolates.

\section{Sulfobacillus abundance and distribution}

Previous data have indicated the presence of Sulfobacillus species in low abundance in late growth stage and submerged biofilms [7], as well as in warmer $\left(\sim 43^{\circ} \mathrm{C}\right)$ areas of the Richmond Mine [6]. They are sometimes found to form compact structures of rope-like assemblages (Additional file 3: Figure S2), and similar filamentous-like growth as been reported elsewhere [1,15]. Metagenomic sequencing data indicated that species AMDSBA1 and AMDSBA4 are most abundant in late-growth stage biofilms (Additional file 4: Figure S3). The higher abundances in the '5way fungal streamer' sample may reflect the unique DNA-extraction procedure (grinding in liquid nitrogen) used on this sample alone, potentially improving lysis of Gram-positives and their vegetative spores.

\section{Energy metabolism: electron transport chain}

Prior studies have shown that Sulfobacillus are capable of aerobic growth. Genomes indicate that oxygen reduction can occur via several different terminal oxidases. Each of the Sulfobacillus genomes analyzed in this study has genes encoding the four-subunit proton pumping quinol oxidases, although only a fragment of subunit II

Table 1 General statistics of the Sulfobacillus genomes

\begin{tabular}{|c|c|c|c|c|c|c|c|}
\hline Name & $\begin{array}{c}\text { Genome size } \\
\text { (Mbp) }\end{array}$ & GC Content (\%) & Coverage $(X)$ & Scaffolds & N50 & $\begin{array}{l}\text { Protein coding } \\
\text { genes }\end{array}$ & Reference \\
\hline AMDSBA1 & 4.56 & 52.19 & 258 & 155 & 65,127 & 4629 & This study \\
\hline AMDSBA2 & 3.07 & 52.37 & 10 & 409 & 10,877 & 3312 & This study \\
\hline AMDSBA3 & 3.68 & 55.54 & 113 & 129 & 49,240 & 3785 & This study \\
\hline AMDSBA4 & 4.11 & 51.67 & 25 & 179 & 63,277 & 4254 & This study \\
\hline AMDSBA5 & 3.65 & 49.15 & 92 & 161 & 65,096 & 3870 & This study \\
\hline $\begin{array}{l}\text { S. thermosulfidooxidans } \\
\text { AT-1 (DSM 9293) }\end{array}$ & 3.86 & 49.64 & N/D & 2 & - & 3875 & $|M G J G|$ \\
\hline S. thermosulfidooxidans 'Cutipay' & 3.86 & 49.30 & 116 & 35 & 509,367 & 3600 & Travisany [16] \\
\hline $\begin{array}{l}\text { S. acidophilus } \mathrm{NAL}^{\top} \\
\text { (DSM 10332) }\end{array}$ & 3.56 & 56.75 & 168 & 1 & - & 3626 & Anderson [17] \\
\hline S. acidophilus 'TPY' & 3.55 & 56.70 & 26 & 1 & - & 3770 & $\mathrm{Li}[2]$ \\
\hline
\end{tabular}



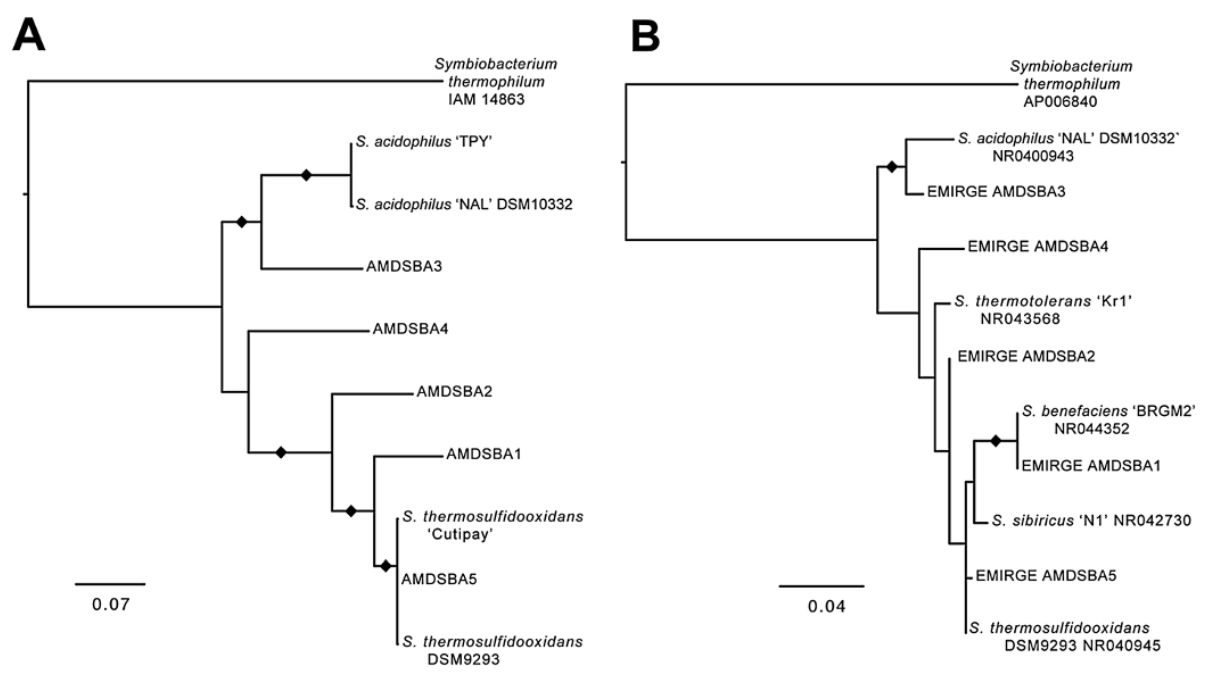

Figure 2 Maximum likelihood phylogenetic trees showing relationship amongst Iron Mountain and published Sulfobacillus species using A) concatenated alignment of sixteen ribosomal proteins and B) EMIRGE-generated 16S rRNA genes. Black diamonds indicate nodes with greater than $90 \%$ bootstrap support.

of the quinol oxidases from AMDSBA4 and AMDSBA2 were obtained. AMDSBA1 and AMDSBA3 contained an additional quinol oxidase (Figure 3, Additional file 5: Table S2), and this second copy may have a different affinity for oxygen. Except for AMDSBA3, all of the Sulfobacillus genomes also contain cytochrome $c$ oxidase encoding-genes. The number of cytochrome $c$ oxidaseencoding genes varies between species with $S$. acidophilus containing 2 copies, with $S$. thermosulfidooxidans strains containing at least 4 (Figure 3). Furthermore, a unique terminal oxidase from AMDSBA4 (AMDSBA4_44_9-12) was orthologous to the quinol oxidases of the other Sulfobacillus (and contained four subunits), however it contained a $\mathrm{Cu}_{\mathrm{A}}$-binding site in subunit II, indicating it accepts electrons from cytochrome $c$ and not quinones, [51,52]. Additionally, cytochrome $c$-binding sites $(\mathrm{CXXCH})$ were identified in the $\mathrm{C}$-terminal end of both subunits II and IV which are not present in any other terminal oxidases here, suggesting the electron transport chain of AMDSBA4 is distinct from other Sulfobacillus species.

AMDSBA4 is also unique in that it contains a cytochrome $b c$ complex. Other Sulfobacillus have a di-heme cytochrome $b$ homologous to the pet $B$ gene found in other cytochrome $b c$ complexes, however the absence of cytochrome $c$ and the iron sulfur protein in these operons suggests that these are not canonical $b c$ complexes and may perform some other function.

c-type cytochromes are annotated in all genomes except AMDSBA1, although the presence of cytochrome $c$ oxidases and cytochrome $c$ assembly factors would indicate that $c$-type cytochromes are likely present.
All of the Sulfobacillus genomes contain multiple genes encoding the two-subunit $b d$-type quinol oxidases. The cytochrome $b d$ respiratory oxygen reductases contribute to the proton motive force by transmembrane charge separation without a proton pump [53].

Each genome contains genes encoding a classic 14subunit NADH:quinone oxidoreductase (Complex 1). In addition, an 11-subunit Complex 1 is conserved in AMDSBA1, AMDSBA2, AMDSBA5, and S. acidophilus. This 11 subunit gene cluster lacks genes encoding the "N-module" subunits (nиоE, nuoF, and nuoG) that are involved in NADH binding, and has been suggested to be the evolutionary ancestor of the 14 subunit complex $[54,55]$. The lack of the $\mathrm{N}$-module suggests it can receive electrons from donors other than NADH [54].

Genes encoding succinate:quinone oxidoreductases (SQORs) are found in all genomes. These SQORs are characterized by having a single hydrophobic subunit anchor with two heme groups, and thus can be classified as 'Type B' [56]. AMDSBA4, however, has an additional SQOR adjacent to the first (AMDSBA4_13_54-57), distinct in that it contains two hydrophobic subunit anchors, each predicted to bind a heme group. As such, it is most similar to 'Type A' SQOR enzymes commonly associated with Archaea [56,57]. The functional significance of this second SQOR in AMDSBA4 is unclear.

\section{Sulfur metabolism}

Oxidation of sulfur, tetrathionate, and sulfide minerals is a well-documented characteristic of Sulfobacillus spp. $[5,10,11,13]$, and the genomes analyzed here are replete with enzymes involved in sulfur oxidation. In the AMD 


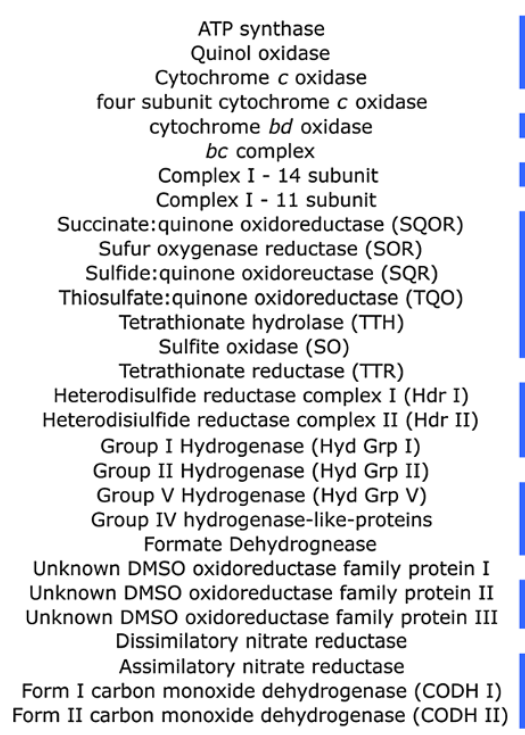

Figure 3 Gene copy numbers for electron transport chain, sulfur, hydrogen, nitrogen metabolisms in Sulfobacillus genomes. A filled blue square indicates presence, with a white number indicating copy numbers $>1$. Filled grey squares are presumed present due to presence in closely related organisms or partial gene identification. Colored circles and triangles at the top of the graph indicate species identifier in Figure 5.

environment, elemental sulfur and various polythionates such as tetrathionate and pentathionate are ultimately formed by the reaction of acid-insoluble sulfide minerals (e.g., pyrite) with ferric iron [58-60].

Aerobically, Sulfobacillus spp. may oxidize sulfur in a disproportionation reaction using sulfur oxygenase reductase (SOR), which disproportionates linear polysulfide in the presence of oxygen to sulfite and hydrogen sulfide [61]. SOR is found in S. acidophilus, AMDSBA2, and in two copies in both S. thermosulfidooxidans and AMDSBA5 (Figure 3, Additional file 5: Table S2). Best studied in the archaeon Acidianus ambivalens [61], the SOR protein is cytoplasmic, and the lack of cofactors means it is not coupled to electron transport, supported by work done in Acidithiobacillus caldus [62]. All Sulfobacillus SOR proteins possess the identified conserved residues for a functional enzyme $[61,63]$.

Another possible route of sulfur compound oxidation may be through heterodisulfide reductase-like (Hdr) proteins encoded in all Sulfobacillus genomes. In methanogens, heterodisulfide reductases reduce heterodisulfide bonds in the final step of methanogenesis [64]. However, proteins related to heterodisulfide reductases are widely distributed and are suspected to be

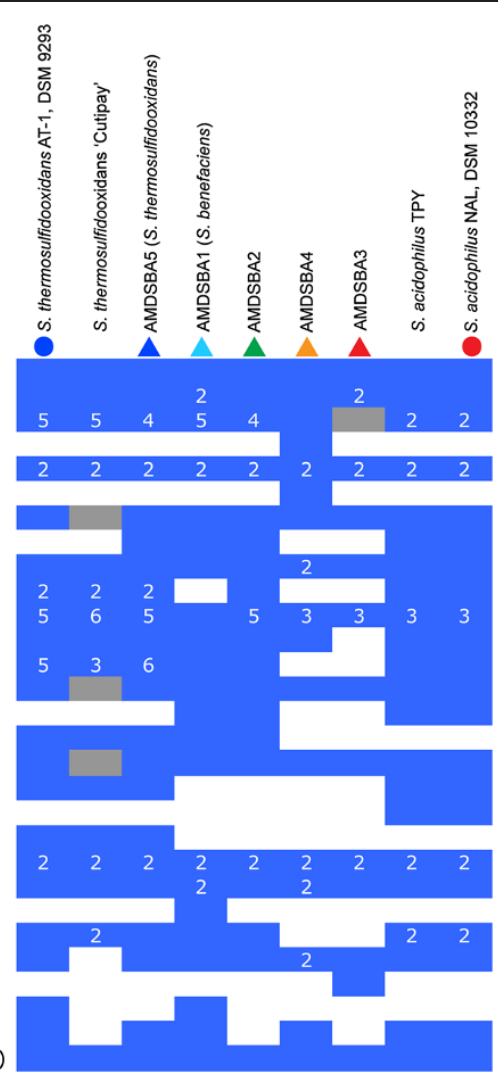

involved more generally in various forms of sulfur metabolism $[65,66]$. Clusters of Hdr-like proteins are conserved in other acidophilic sulfur oxidizing Bacteria and Archaea and are upregulated during aerobic growth on sulfur in Aciditothiobacillus ferroxidans, where they are predicted to oxidize disulfide intermediates to sulfite [67]. The proposed disulfide substrates are sulfane sulfur species $\left(\mathrm{RSS}_{\mathrm{n}} \mathrm{H}\right)$, formed by the reaction of cellular thiols (e.g., cysteine residues) with extracellular elemental sulfur $[68,69]$.

Two different types of Sulfobacillus hdr gene clusters were found with varying gene content (Figure 4). One group, $h d r$ gene cluster I, was identified only in S. thermosulfidooxidans, AMDSBA1 and AMDSBA5. This cluster includes an HdrA-like protein with an FAD-binding site and a conserved-cysteine motif $\left(\mathrm{CxGCRDx}_{6-8} \mathrm{CSx}_{2} \mathrm{CC}\right)$ typical in binding an $\mathrm{Fe}-\mathrm{S}$ cluster. Two HdrB proteins are present which contain the cysteine-rich CCG domains $\left(\mathrm{Cx}_{\mathrm{n}} \mathrm{CCGx}_{\mathrm{m}} \mathrm{C}\right)$ predicted to bind [4Fe-4S] clusters [70]. The HdrC protein contains another [4Fe-4S] binding center. Furthermore, two DsrE-family proteins and a TusA homolog are encoded in this locus. DsrE and TusA proteins are strongly implicated in persulfidic sulfur (RS-SH) transfer in Allochromatium. vinosum [71,72]. It is also 


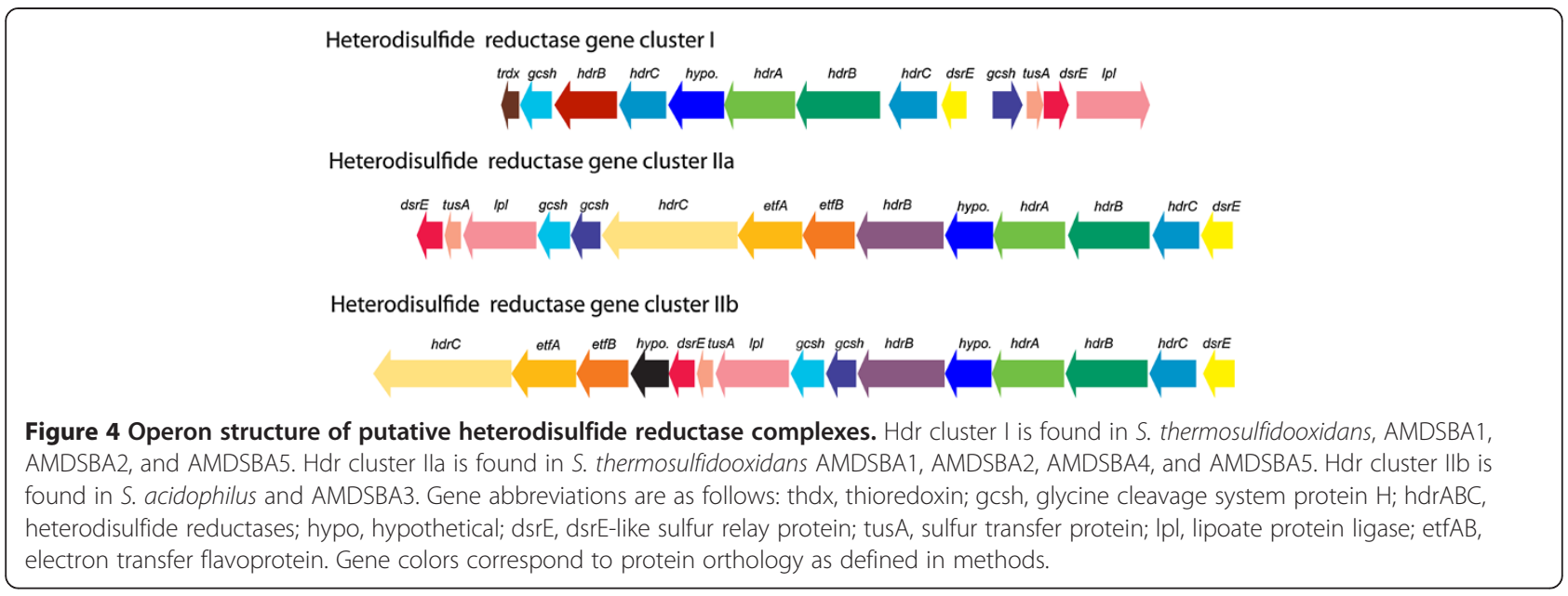

interesting to note that a homolog to the glycine cleavage protein $\mathrm{H}$ (a lipoic acid containing protein) is found in this locus as is a lipoate-protein ligase that catalyzes the attachment of lipoic acid to target proteins [73]. The lipoic acid moiety of glycine cleavage protein $\mathrm{H}$ acts as a "swinging arm" that serves to transfer reaction intermediates between the various catalytic sites of the other proteins in the glycine cleavage system complex [74]. It is tempting to speculate that lipoic acid, with its disulfide bond, may play a key role in sulfur species transfers amongst this heterodisulfide complex, as has been noted by others [75].

The second group ( $h d r$ gene cluster II) is found in all Sulfobacillus genomes and contains many of the same subunits as $h d r$ gene cluster I (Figure 4). However, there are additional genes encoding electron transfer flavoproteins as well as a second, longer $h d r C$ gene containing 6 transmembrane helices and 2 cysteine-rich CCG domains not found in the $\mathrm{HdrC}$ of $h d r$ gene cluster I. These three genes are syntenous, and similar arrangements have been observed in the sulfate reducing bacterium Desulfobacterium autotrophicum [76].

After SOR catalyzes the disproportionation reaction of linear polysulfide to $\mathrm{H}_{2} \mathrm{~S}$ and sulfite, the sulfide may be oxidized back to sulfur by the membrane-bound sulfide: quinone oxidoreductases (SQR), with the concomitant reduction of quinones. Thus, sulfur disproportionation might be linked to the electron transport chain $[77,78]$. Across the Sulfobacillus genomes, 74 proteins were annotated as SQRs or more generally as "FAD-dependent pyridine nucleotide disulfide oxidoreductases," the family to which SQR proteins belong. There are three active-site cysteine residues shown to be important for SQR activity, with the second and third being essential $[77,79]$. Alignment of these 74 Sulfobacillus putative SQRs and their related oxidoreductases with the characterized SQR of Acidothiobacillus ferroxidans showed 9 as having all three active-site cysteine residues, and 27 as having the second and third essential active-site cysteine residues.
Protein trees constructed with those 36 Sulfobacillus proteins containing at least the second and third essential active-site cysteine residues indicated that all proteins belonged to either Group III or Group V SQRs, based on the protein phylogeny laid out by Marcia et al. [77] (Additional file 6: Figure S4). Both groups have representatives with demonstrated sulfide:oxidase activities $[77,80]$. Based on this analysis, SQR is likely present in the published genomes of all Sulfobacillus species analyzed here.

Tetrathionate can be disproportionated by tetrathionate hydrolase (TTH) into sulfate, thiosulfate, and other sulfur compounds [81-83]. TTH activity has been detected in a strain of S. thermosulfidooxidans [84], and tetrathionate oxidation is widespread amongst Sulfobacillus isolates [85]. TTH homologs were found in all but AMDSBA3 of the Sulfobacillus genomes (Figure 3, Additional file 5: Table S2). Based on TTH-family phylogeny laid out by Protze et al. [86], only S. thermosulfidooxidans, S. acidophilus, and AMDSBA1 have proteins that fall within characterized TTH-family clades (Additional file 7: Figure S5). The remaining enzymes belong to yet uncharacterized families of TTH homologs. Furthermore, TTH proteins have been shown to be periplasmic in Gram-negative organisms [[83] and refs therein], and thought to be extracellular in the archeaon Acidianus ambivalens [86], and thus may also be extracellular in Sulfobacillus species.

Membrane-associated thiosulfate:quinone oxidoreductase (TQO) enzymes were found in all Sulfobacillus genomes analyzed except for AMDSBA3 (Figure 3, Additional file 5: Table S2). TQO may oxidize thiosulfate formed by TTH back to tetrathionate and concomitantly passing electrons into the electron transport chain via the quinone pool [87].

Another mechanism of tetrathionate metabolism could occur via putative tetrathionate reductases (TTR), an enzyme belonging to the dimethylsulfoxide oxidoreductase (DMSOR) family of proteins with a molybdobis-pyranopterin guanine-dinucleotide cofactor [88]. 
Several Sulfobacillus DMSOR catalytic subunits clustered with a branch containing tetrathionate reductases, including the characterized tetrathionate reductase from Salmonella enterica (Additional file 8: Figure S6). In S. enterica, TTR is a three-subunit enzyme that can reduce trithionate and tetrathionate to thiosulfate and sulfite [89]. TTR proteins were found in S. acidophilus, AMDSBA1, and a partial sequence was available from AMDSBA2. Except for a second putative TTR in AMDSBA1 (AMDSBA1_3_25, which also lacks the second and third subunits), the putative TTR first and second subunits each contain a Tat-signal sequence, indicating an extracellular location. No obvious heme or quinonebinding subunits were observed in the membraneanchor subunit, leaving questions as to how, or if, this enzyme interacts with an electron transport chain of the cell. Furthermore, we note that no tetrathionate reducing activity has been observed for any Sulfobacillus species, and it is impossible to say what the function of this enzyme is based on genomic inference alone at this time.

Other catalytic subunits of Sulfobacillus DMSOR-family proteins clustered in a branch containing the sulfur reductase of Aquifex aeolicus (SreA) as well as the sulfiteoxidizing protein of Allochromatium vinosum (SoeA, Additional file 8: Figure S6). The three-subunit sulfur reductase complex in $A$. aeolicus is suggested to be involved in the reduction of tetrathionate, sulfur, and polysulfide coupled to $\mathrm{H}_{2}$ oxidation via a quinone pool [90]. On the other hand, the three-subunit sulfiteoxidizing complex in $A$. vinosum couples quinone reduction to sulfite oxidation [91]. Similar three-subunit gene operons are found in all Sulfobacillus species analyzed here, and as no sulfur-compound reduction has yet been observed for any Sulfobacillus species, we believe that the Sulfobacillus proteins are likely sulfiteoxidizing proteins akin to the SoeABC proteins of A. vinosum. The Sulfobacillus proteins lack signal peptides, and thus, like the SoeABC complex in $A$. vinosum, may be cytoplasmically located and anchored to the membrane.

Sulfate assimilation may occur in S. thermosulfidooxidans, S. acidophilus, AMDSBA1, and AMDSBA3 by the combined action of an ATP sulfurylase, an adenosine 5'-phosphosulfate (APS) reductase, a ferredoxin-dependent sulfite reductase, and an $\mathrm{O}$-acetylserine sulfhydrolase. The APS-reductase is homologous to 3'phosphoadenosine 5'-phosphosulfate (PAPS) reductases often found in sulfate assimilation pathways, but the presence of an iron-sulfur binding subunit suggests the preferred substrate is APS [92,93]. AMDSBA5 lacked the ATP sulfurylase and APS reductase, thus inorganic sulfur assimilation may proceed from sulfite. Furthermore, AMDSBA2 and AMDSBA4 did not contain the sulfite reductase, which may reflect incomplete genomic coverage or point to inorganic sulfur assimilation directly from $\mathrm{H}_{2} \mathrm{~S}$.

\section{Iron oxidation and reduction}

Many Sulfobacillus isolates are demonstrated iron oxidizers $[1,10,11,13]$. In other acidophilic microorganisms, outer-membrane cytochromes have been implicated in the oxidation of iron, including in the Gram-negative bacteria Leptospirillum spp. and Acidothiobacillus ferroxidans [[94] and refs therein]. Electrons from iron are ultimately transferred to either a terminal oxidase complex to reduce oxygen and consume and pump protons ("downhill") or to an NADH dehydrogenase ("uphill") [94]. There are membrane-associated $c$-type cytochromes that could be involved in iron oxidation in all Sulfobacillus species examined here except AMDSBA1. Electrons could be transferred from these membrane $c$-type cytochromes to the terminal cytochrome $c$ oxidases in a "downhill" oxidation of iron. The "uphill" reactions require harnessing the proton-motive force in order to push the electrons in a thermodynamically unfavorable direction, and this is accomplished by the $b c$ complex in Leptospirillum spp. and A. ferroxidans [94]. Only AMDSBA4 is predicted to have this complex, and it would be interesting to know if it functions in reverse electron transport as in the Gram-negative iron oxidizers. Furthermore, genes encoding putative sulfocynanin proteins have been identified in a strain of $S$. thermosulfidooxidans (S. thermosulfidooxidans ST) and shown to be highly expressed during growth on ferrous sulfate [18]. The extent to which these may be involved in iron oxidation is unclear.

All isolate species of Sulfobacillus have been shown to reduce ferric iron $[10,11,13,14]$. Although $\mathrm{Fe}^{3+}$ reduction is widespread in acidophilic microorganisms [95,96], little is known about the precise enzymes and pathways involved. In $A$. ferroxidans, a $c$-type cytochrome has been implicated in ferric iron respiration [97] as has a rusticyanin [98]. Rusticyanin proteins are found annotated only in $S$. thermosulfidooxidans and AMDSB5, and membrane-bound $c$-type cytochrome proteins are found in all Sulfobacillus genomes except AMDSBA1. Osorio et al. [99] also proposed a model for ferric iron reduction in $A$. ferroxidans when elemental sulfur was an electron donor, including one in which $\mathrm{Fe}^{3+}$ was indirectly reduced by $\mathrm{H}_{2} \mathrm{~S}$ generated from anaerobic sulfur disproportionation. This $\mathrm{H}_{2} \mathrm{~S}$ 'scavenging' allows normally endergonic sulfur disproportionation to become thermodynamically favorable [100,101]. While generation of hydrogen sulfide may play a role in ferric iron reduction, Sulfobacillus have shown to reduce iron in cultures without any sulfur species present [13,102], implying that some mechanism for the direct reduction of iron is also present.

\section{Hydrogen metabolism}

The catalytic subunits for twenty-seven different nickeliron $(\mathrm{NiFe})$ hydrogenase proteins were identified across 
all Sulfobacillus species, which cluster into 5 distinct subfamilies (Additional file 9: Figure S7). One conserved 16-subunit gene cluster was identified within S. acidophilus, S. thermosulfidooxidans, and AMDSBA5, and the catalytic subunit was found to cluster with Group 1 respiratory-uptake [NiFe]-hydrogenases (Additional file 9: Figure S7). Like other Group 1 hydrogenases, the Sulfobacillus hydrogenases possess a Tat-motif at the N-terminus of the small subunit and are thus predicted to be located extracellularly. There, they may oxidize $\mathrm{H}_{2}$, transferring protons to the quinone pool via a cytochrome $b$-containing subunit [103]. The second 16-subunit hydrogenase gene cluster was identified in S. acidophilus and S. thermosulfidooxidans species, although the operon structure and catalytic subunits differ (Additional file 5: Table S2). In S. acidophilus, the catalytic subunits share the greatest homology with subgroup A of the Group 2 hydrogenases (Additional file 9: Figure S7). Group 2 hydrogenases are cytoplasmic hydrogen-utilizing complexes most commonly associated with nitrogen fixing cyanobacteria [103]. The hydrogenases of $S$. thermosulfidooxidans, however, are most similar to a relatively recently identified fifth group of hydrogenases [Additional file 9: Figure S7, [104]]. This fifth group is composed of putative high-affinity hydrogenases and has been detected predominantly in Actinobacteria, Acidobacteria, and Chloroflexi [105]. Neither the hydrogenases of Group 2 nor Group 5 contain a signal peptide, and are thus predicted to remain in the cytoplasm. Moreover, they do not contain the $b$-type cytochrome subunit thought to aid in electron transfer to quinones, and the electron acceptor is unclear. No Group 1, 2 or 5 hydrogenases were found in AMDSBA1-4.

The catalytic subunits of the remaining hydrogenases indicate that they belong to the Group 4 hydrogenase family, and at least one representative is found in each Sulfobacillus species (Additional file 9: Figure S7). Six syntenic genes are found near each catalytic subunit (Additional file 5: Table S2), and bear homology to Complex I enzymes (nuoH, nuoL) and formate hydrogen-lyase enzymes (HyfE and HyfB). The catalytic large subunits of these hydrogenases lack the binding site motifs normally found in [NiFe] hydrogenases [N-terminal: $\mathrm{RxCGxCxxxH}$; C-terminal DPCxxCxxH/R; [106]]. As such, these enzymes are similar to energy-converting hydrogenase-related complexes (Ehr) and their function remains unknown $[103,107,108]$.

\section{Carbon monoxide dehydrogenases}

Aerobic-type carbon monoxide dehydrogenases (CODH) of both Form 1 and Form 2 were identified in Sulfobacillus genomes (Figure 3, Additional file 5: Table S2). Form 1 CODHs are known to be involved in carbon monoxide oxidation to $\mathrm{CO}_{2}$ with release of reducing equivalents introduced into the electron transport chain usually via cytochromes [109]. Form I enzymes were found in all organisms except AMDSBA2 and AMDSBA3. The function of Form II enzymes-differentiated by the active site motif AYRGAGR in place of the Form I motif AYXCSFR-are not fully understood, and may also play roles in $\mathrm{CO}$ oxidation as well as oxidation of other substrates [109]. Form II CODH enzymes are found in all Sulfobacillus species.

\section{Nitrogen metabolism}

AMDSBA3 is the only Sulfobacillus species analyzed here that contains genes for dissimilatory nitrate reduction, observed as a single NarGHJI operon (Additional file 8: Figure S6, Figure 3). No other components for denitrification or nitrite reduction were found. AMDSBA3 is thus the only Sulfobacillus species analyzed here predicted to be capable of using nitrate as an alternative electron acceptor in anaerobic environments.

AMDSBA1 and S. thermosulfidooxidans contained genes encoding assimilatory nitrate reductases (Figure 3, Additional file 5: Table S2). The proteins encoded by the nitrate reductase genes (NasC) are members of the DMSOR family of enzymes, and phylogenetically cluster near the assimilatory nitrate reductase of Bacillus subtilis (Additional file 8: Figure S6). However, unlike the nitrate reductase of $B$. subtilis, the nitrate reductases in AMDSBA1 and S. thermosulfidooxidans do not possess an electron transfer subunit (NasB) required for the transfer of electrons from NADH to nitrate [110], making the electron donor unclear (e.g., NADH, flavodoxin, or ferredoxin). Nearby genes encoding nitrite reductases (NasDE) in both species contain the necessary siroheme and NADH binding sites required for the six-electron reduction of nitrite to ammonia using NADH as the electron donor $[110,111]$. Copper-containing nitric oxide-forming nitrite reductases were found in S. acidophilus strains, as were nitric oxide dioxygenase enzymes, which may oxidize nitric oxide to nitrate as a detoxification mechanism [112]. It has also been suggested that these enzymes may reduce nitric oxide to nitrous oxide anaerobically, which if true, would allow denitrification activity (nitrite to nitrous oxide) in these organisms [113].

Ammonium transporters were identified in all genomes, and ammonia can be assimilated into central metabolic pathways via glutamate dehydrogenase, glutamine synthetase and glutamate synthase.

\section{Autotrophy}

Sulfobacillus spp. are known autotrophs, and published genomes of S. thermosulfidooxidans DSM 9293, and $S$. acidophilus indicate the presence of carbon fixation via Calvin-Benson-Bassham (CBB) cycle $[2,16,17,114]$. 
All Sulfobacillus also have complete CBB cycle genes, including a Type I ribulose-1,5-bisphosphate carboxylaseoxygenase (RuBisCO). In addition to the Type I RuBisCO, AMDSBA4 and S. acidophilus strains have additional Type 4 RuBisCO-like proteins (Additional file 10: Figure S8) that likely participate in the methionine salvage pathway [115]. Interestingly, AMDSBA4 is the only genome with an annotated carboxysome (AMDSBA4_48_6-9).

\section{Central carbon pathways}

In addition to autotrophic growth, Sulfobacillus isolates have been reported to grow mixotrophically and heterotrophically on a variety of carbon substrates, including glucose, fructose, glycerol and other various organic carbon compounds $[11,14,116]$. All organisms except S. thermosulfidooxidans, AMDSBA5, and AMDSBA1 have a complete Embden-Meyerhoff pathway for glycolysis. S. thermosulfidooxidans, AMDBA5, and AMDSAB1, lack a 6-phosphofructokinase. While genes encoding 6-phosphofructokinase could not be identified in $S$. thermosulfidooxidans, cell-free enzymatic assays suggest these cells can carry out this transformation [102]. Glucose-6-phosphate isomerase activity in AMDSBA4 and both S. acidophilus can be accounted for by the inclusion of unique bifunctional transaldolases/phosphoglucose isomerase [117]. The oxidative portion of the pentose phosphate pathway (glucose dehydrogenase, 6-phosphogluconolactonase, and 6-phosphogluconate dehydrogenase) was present in all organisms.

Complete semi-phosphorylative Entner-Doudoroff carbon degradation pathway (2-keto-3-deoxygluconate 6-phosphate aldolase, 2-dehydro-3-deoxygluconokinase, and gluconate dehydratase) was present in S. acidophilus strains, AMDSAB1, AMDSBA3, and AMDSBA4 (Additional file 5: Table S2). While no gluconate dehydratases were annotated, deoxy-acid dehydratases likely carry out this function as they do in other bacteria [118]. Phosphogluconate dehydratases, indicative of the phosphorylative Entner-Doudoroff pathway, were not found in any genomes. Enzyme assays from cell-free extracts of $S$. thermosulfidooxidans and S. sibiricus strains have shown this phosphogluconate dehydratase activity, however, as well as keto-3-deoxy-6-phosphogluconate aldolase activity $S$. thermosulfidooxidans, which would indicate a phosphorylative Entner-Douroroff pathway [102,119].

Pyruvate can be oxidatively decarboxylated to acetylCoA with pyruvate dehydrogenase (found in all Sulfobacillus) or in AMDSBA1 with a putative pyruvate ferredoxin oxidoreductase (AMDSBA1_57_11-13). Excepting a few gaps from the low-coverage genome of AMDSBA2, the tricarboxylic acid cycle was complete in all genomes. Furthermore, a putative oxoglutarate synthase that may allow oxidations of 2-oxoglutarate to be coupled to the reduction of ferredoxin was found in all Sulfobacillus genomes. Finally, a complete glyoxylate bypass system (malate synthase and isocitrate lyase) was found in only AMDSBA1 and S. acidophilus, possibly permitting carbon assimilation of $\mathrm{C}_{2}$ compounds like acetate. Malate synthase was found in all but AMDSBA3 and AMD$\mathrm{SAB} 4$, which together with glycolate oxidase, may provide a route of glycolic acid assimilation. Glycolic acid is produced as an exudate by key primary producing acidophiles like Leptospirillum, and the degradation of glycolic acid sets Sulfobacillus apart amongst other acidophiles. In fact, it is thought to contribute to an organiccarbon degrading niche in stirred-tank bioleaching systems [120]. Lactate utilization proteins similar to those found in Bacillus subtilis were identified in all organisms, and are thought to allow growth on lactate [121].

\section{Formate dehydrogenase}

Putative cytoplasmic selenocysteine-containing formate dehydrogenases are found in all Sulfobacillus genomes (Additional file 8: Figure S6). These genes possess the binding site and three catalytic residues necessary for formate oxidation. Each catalytic subunit is syntenous to cluster of genes with homology to the NADH-binding NuoEFG subunits, and thus may represent coupling of formate oxidation to NADH reduction.

\section{Fermentative metabolism}

Strains of S. thermosulfidooxidans, S. sibiricus, and S. thermotolerans have been shown to produce propionate and acetate in the growth media during growth in hypoxic conditions [15]. Complete fermentative pathways that result in propionate formation, however, could not be identified. In all Sulfobacillus species, neither the enzymes lactoyl-CoA dehydratase [of the acrylate pathway [122]] nor propionyl-CoA:succinate-CoA transferase and methylmalonyl-CoA carboxyltransferase [of the succinate pathway for propionate fermentative pathway [123]] could be identified. Acetate production could be produced with substrate level phosphorylation by a phosphate acetyltransferase (found only in AMDSBA2) and an acetate kinase (identified in AMDSBA1 and AMDSBA2). Traditional fermentative pathways thus cannot account for propionate and acetate produced in isolate strains.

\section{Lipid and lipopolysaccharide biogenesis}

All Sulfobacillus species have the genes required to synthesize fatty acids. Species of Sulfobacillus have been shown to contain predominantly branched-chain anteiso and $\omega$-alicyclic fatty acids [124]. In general, branched chain fatty acids can be synthesized from either branched short-chain carboxylic-acid CoA esters or from $\alpha$-keto acids [125]. In the Sulfobacillus, the former pathway is 
probably used, as branched chain $\alpha$-keto acid decarboxylases were not identified. The chorismate pathway and genes encoding biosyntheis of $\omega$-alicyclic fatty acids were identified in all Sulfobacillus species. The chorismate pathway produces shikimic acid as an intermediate [126], which is ultimately used to form cyclic carboxylic acid-coA esters used in the synthesis of $\omega$-alicyclic fatty acids. Peptidoglycan biosynthesis was accounted for in all Sulfobacillus. An S-layer has been reported to be present on some cells of $S$. thermosulfidooxidans [127], however only a single annotated S-layer protein was found in AMDSBA1 (AMDSBA1_76_63). Notably, a gene cluster present only in AMDSBA3 encodes for a putative squalene biosynthetic pathway (AMDSBA3_32_15-22), including a squalene-cyclase that could be involved in the production of hopanoids $[128,129]$. Hopanoids have been previously identified in acidophilic organisms [130], and are thought to alter membrane fluidity and permeability to protons [131].

\section{Vitamin and coenzyme biosynthesis}

Menaquinone biosynthetic pathways were identified in all Sulfobacillus genomes, in agreement with detection of a menaquinone with 7 isoprenoid units in Sulfobacillus thermotolerans [11]. Complete biosynthetic pathways for lipoic acid, coenzyme A, nicotinamide adenine dinucleotide, flavin adenine dinucleotide, and biotin were identified. Vitamin B12 biosynthetic proteins were found in all organisms except AMDSBA3 and AMDSBA4. All Sulfobacillus genomes have B12-dependent methylmaloynl-CoA genes, and thus all likely require the B12 cofactor. The corrinoid-transporting enzyme encoded by the $b t u F$ gene was found in all species, thus AMDSB3 and AMDSBA4 probably scavenge vitamin B12 from the environment while the other Sulfobacillus can either scavenge or synthesize B12 de novo.

\section{Environmental stress}

Genes encoding alkyl hydroperoxide reductases/peroxiredoxins and superoxide dismutases were found in all species. Oxidative stress responses are important in all aerobic Bacteria and Archaea, and defense against reactive oxygen species in metal-rich acidic environments is especially important due to redox-active metals creating reactive-oxygen species via the Fenton reaction [132]. Thioredoxin reductases were also identified and may play an important role in reactive oxygen defense as identified in acidophilic Leptospirillum bacteria [133].

Genes encoding for the biosynthetic pathways of ectoine and hydroxyectoine were found solely in AMDSBA1 (AMDSBA1_21_55-58). In all Sulfobacillus, trehalose could be synthesized by the combined actions of trehalose- 6 phosphate synthase and trehalose-6-phosphate phosphatase. However, a second trehalose biosynthetic pathway, involving trehalose synthase [134], was found in S. thermosulfidooxidans as well as AMDSBA1, AMDSBA2, and AMDSBA5. Compatible solutes play important roles in adaptation to the osmotic stress induced by the highionic strength environments like those found in metalrich, acidic environments.

All Sulfobacillus isolates are known spore formers $[10,11,13]$, and all genomes analyzed here are consistent with sporulation capability.

\section{Transport}

Transporters for citrate, potassium, phosphate were identified in all species as were chromate, copper, and arsenite efflux pumps. Numerous amino acid permeaselike proteins, oligopeptide and dipeptide transporters, and sugar $A B C$ transport proteins were identified in all species, consistent with a heterotrophic mode of carbon assimilation. Polar amino acid $\mathrm{ABC}$ transporters were found only in AMDSBA1, AMDSBA4, and both S. acidophilus species and branched amino-acid transport systems were found in all but AMDSBA3. A putative taurine transporter was identified in all but AMDSAB3 and AMDSBA4. Taurine has been identified as a highabundance compatible solute putatively synthesized by Eukaryotes in the Richmond Mine AMD system, and may provide an important source of nitrogen, carbon, and sulfur for Sulfobacillus organisms [135].

\section{Motility}

All Sulfobacillus contained operons for the synthesis of flagella and chemotaxis signal transduction systems. Notably, both $S$. acidophilus strains contain nine separate methyl-accepting chemotaxis proteins involved in the transduction of environmental signals to motility response [136]. The other genomes contained far fewer, with AMDSBA2 containing six; AMDSBA3 containing four; and S. thermosulfidooxidans, AMDSBA5, AMDSBA4 and AMDSBA1 all containing two or less.

\section{Discussion}

Analysis of the Sulfobacillus genomes illuminates metabolic pathways used in various transformations of carbon, nitrogen, and sulfur metabolism (summarized Figure 5 and Additional file 5: Table S2). Moreover, potential new metabolisms not yet observed in isolate cultures are potentially encoded within these genomes, including hydrogen oxidation and nitrate reduction.

Carbon fixation via the Calvin cycle is a well-described characteristic of Sulfobacillus species [114], and type I RuBisCO genes were identified in all Sulfobacillus genomes. It has been noted that Sulfobacillus growth rates increase when they are cultivated in the presence of yeast extract and simple sugars [102,137]. Optimum growth under mixotrophic conditions using inorganic 


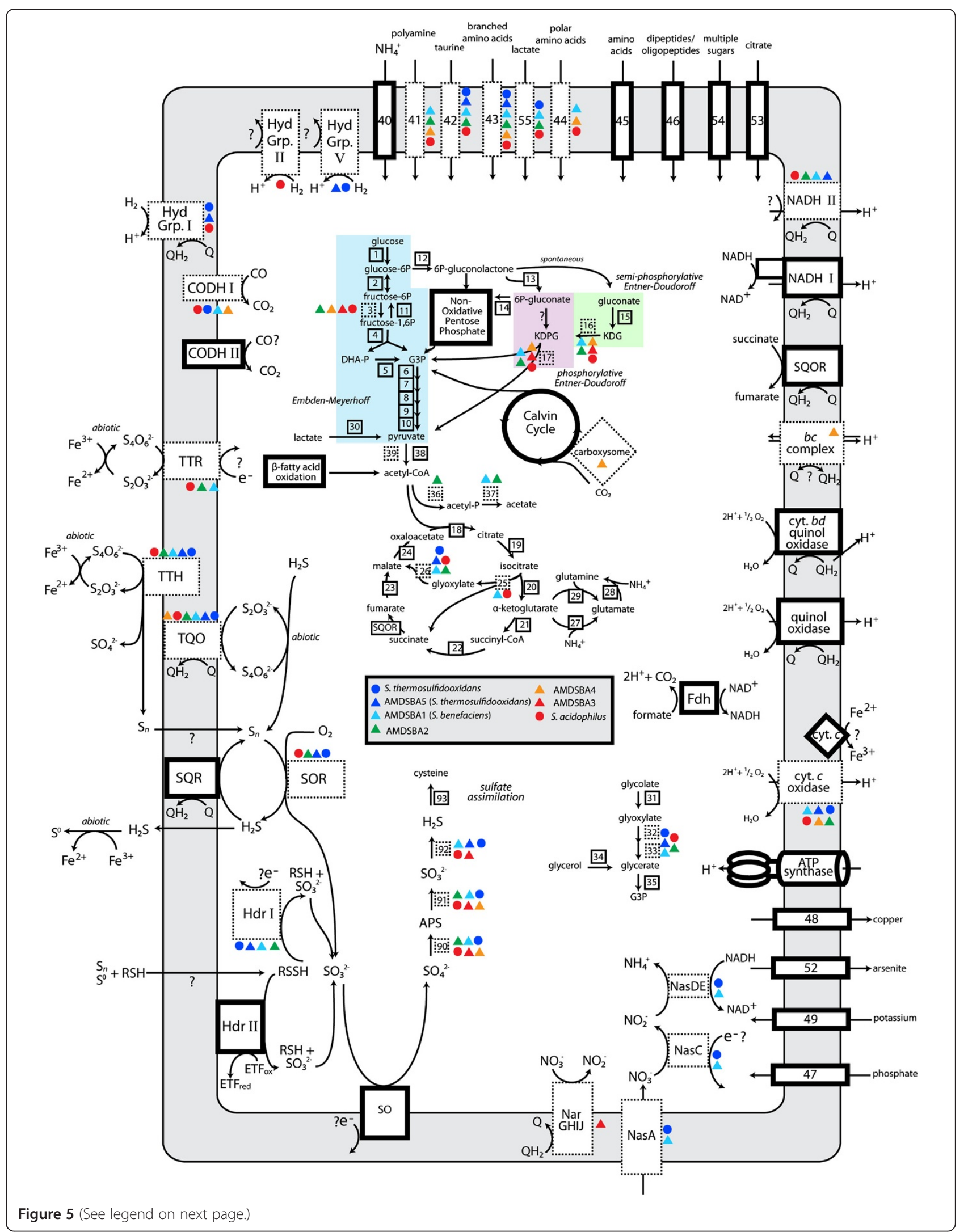


(See figure on previous page.)

Figure $\mathbf{5}$ Reconstruction of central metabolism of Sulfobacillus species. Dark bordered boxes represent enzymes or enzyme complexes found in all species, while boxes bordered by dashed lines represent those present in only a subset. Numbers correspond to genes listed in Additional file 5: Table S2. Colored circles (previously published genomes) and triangles (AMDSBA genomes) represent which organisms those enzymes are found in, as indicated in the figure legend. $\mathrm{CODH}$, carbon monoxide dehydrogenase complexes; DHA-P, dihydroxyacetone phosphate; Fdh, formate dehydrogenase; G3P, glyceraldehyde-3-phosphate; Hdr, heterodisulfide reductase complexes; KDG, 2-keto-3-deoxygluconate; KDPG, 2-keto-3-deoxygluconate 6-phosphate; SQR, sulfide:quinone oxidoreductae; SQOR, succinate:quinone oxidoreductase; SO, sulfite oxidase; TTH, tetrathionate hydrolase; TQO, thiosulfate:quinone oxidoreductase; TR, tetrathionate reductase.

electron donors (usually ferrous iron) and organic carbon (usually glucose or yeast extract) is widely observed [102,138-140]. To our knowledge, only one isolated strain (S. acidophilus NAL) is capable of purely heterotrophic growth, although growth rates are greatly decreased [1]. In another strain of $S$. acidophilus (ALV), it was estimated that about $20 \%$ of cellular carbon was derived from $\mathrm{CO}_{2}$ fixation during growth on glucose [139].

Prior work demonstrated that glucose consumption proceeded via the oxidative pentose phosphate pathway [139], and that enzymes of the Entner-Doudoroff pathway (6-phosphogluconate dehydratase and 2-keto-3deoxy-6-phosphogluconate aldolase) were not present. While our results are consistent with the absence of 6-phosphogluconate dehydratase in all genomes studied here, the 2-keto-3-deoxy-6-phosphogluconate aldolase was found in several AMDSBA genomes as well as in the genomes of $S$. acidophilus strains TPY and NALallowing for a complete semi-phosphorylative EntnerDoudoroff pathway. The presence of this enzyme in $S$. acidophilus NAL may be a key component contributing to its ability to grow heterotrophically.

Across all genomes analyzed here, only two of the enzymes involved in sulfur oxidation were conserved: sulfide: quinone oxidoreductases (SQR) and the heterodisulfide reductase cluster II (Hdr II). While the Hdr II proteins were only present in a single copy amongst all genomes, the SQR proteins were found in between three and six copies for all organisms except AMDSBA1, which contained only one copy (Figure 3). Although the precise reason for the multiple copies of the SQR is unclear, it suggests the importance of sulfide oxidation for many of these organisms.

Sulfide, along with sulfite, can be formed aerobically through a disproportionation reaction of elemental sulfur with sulfur oxygenase (SOR). The subsequent oxidation of sulfide by sulfide:quinone reductase (SQR) allows for the disproportionation reaction of SOR to be coupled to the electron transport chain. While all organisms contain SQR proteins, AMDSBA1, AMDSBA3 and AMDSBA4 lack the SOR proteins necessary to disproportionate sulfur. For these organisms, the source of hydrogen sulfide is unclear, although it may be derived from anaerobic sulfur disproportionation. In this pathway, electrons from sulfane oxidation at the heterodisulfide reductase complex could be used to reduce sulfur, as was proposed for A. ferroxidans [99].

Hydrogen utilization, to our knowledge, has also never been experimentally observed in any Sulfobacillus species. The presence of several different uptake hydrogenase complexes, however, suggests that hydrogen may be an important source of low-potential electrons for these organisms. In the AMDSBA genomes, only AMDSBA5 contained hydrogenases and these complexes may be a key component of niche specialization for AMDSBA5 in the Richmond Mine. The source of hydrogen, however, is unclear, although flammable and odorless bubbles of gas have been observed to be trapped within the biofilms found in the mine (unpublished observations).

Thiosulfate has also been detected in sediments as well as in solutions within the Richmond Mine [141]. Thiosulfate is predicted to be the first aqueous reaction product of pyrite oxidation $[8,60]$. However, because thiosulfate is readily oxidized by ferric iron in acidic media, it is predicted to be found only on the surface of pyritic sediments in the presence of ferric iron. Indeed, thiosulfate was found in bulk solution only in highly reduced conditions essentially devoid of $\mathrm{Fe}^{3+}$ [141]. The availability of thiosulfate may explain why Sulfobacillus spp. are found so infrequently in the floating biofilms of the Richmond Mine, and are sometimes associated with the sediment or as members of submerged biofilms [7]. Tetrathionate, formed by the oxidation of thiosulfate by ferric iron, is far more stable at acidic $\mathrm{pH}$ [142], and thus it is surprising that it was not detected in AMD solutions in the Richmond Mine [8]. The absence of tetrathionate, however, can be explained if it is rapidly oxidized by Sulfobacillus organisms.

Amongst all of the genomes analyzed here, AMDSBA4 is unique in many respects. In addition to the putative carboxysome proteins, AMDSBA4 possesses the only $b c$ complex amongst all of the Sulfobacillus. This is significant as the $b c$ complex has been implicated in reverse electron transport during aerobic iron oxidation in Acidothiobacillus and Leptospirillum species [94]. Furthermore, a second succinate dehydrogenase and a unique cytochrome $c$-oxidase suggest that there may be many distinct aspects of electron transport in AMDSBA4 that warrant further investigation. 


\section{Conclusions}

The genomic comparisons presented here advance our understanding of the metabolic potential within the genus Sulfobacillus and demonstrate diverse ecological strategies for these organisms. Pathways involved in inorganic sulfur, hydrogen, and nitrogen metabolisms are distributed unevenly amongst species, suggesting key differences in energy metabolism that may be foundational to niche differentiation. Common to all Sulfobacillus genomes, however, is a high degree of metabolic versatility, enabling these species to survive in a wide range of acidic environments and adapt to changing biogeochemical conditions. Information gained from this study will help unravel the ecological function of these organisms and their interactions with other organisms in both natural and industrial environments.

\section{Availability of supporting data}

Raw reads were deposited in the NCBI Sequence Read Archive under accession number SRA:SRR191843. Genomes are available at http://ggkbase.berkeley.edu/.

\section{Additional files}

Additional file 1: Figure S1. Genome completeness as estimated by the number of conserved single copy genes identified in each of the Sulfobacillus genomes.

Additional file 2: Table S1. $16 \mathrm{~S}$ rRNA gene similarity (across 1,124 aligned positions) for published and reconstructed Sulfobacillus species. Note that AMDSBA 16S rRNA gene sequences are EMIRGE-generated.

Additional file 3: Figure S2. FISH images of AB Muck submerged biofilm showing Sulfobacillus (SUL230 probe, red) and archaeal (ARC15 probe, green) populations.

Additional file 4: Figure S3. Sulfobacillus abundance estimated as a percentage of basepairs mapped to each organism over total sequenced DNA in each sample. 5 way fungal streamer data is removed in (B) to better visualize low-abundance organisms. Sample and growth stage is depicted on the X-axis, with GSO-1 indicating low growth-stage biofilms, and GS1.5-2 indicating more mature, thicker growth stage biofilms.

Additional file 5: Table S2. Gene loci for central metabolisms discussed in text for all Sulfobacillus species. Column "number on figure" refers to numbers indicated in metabolic reconstruction in Figure 5.

Additional file 6: Figure S4. Phylogenetic analysis of sulfide:quinone oxidoreductases proteins (SQR). Sequences from Sulfobacillus genomes are listed in red. Diamonds indicate nodes with $>90 \%$ bootstrap support. Bootstrap values greater than $55 \%$ are shown as text. Asterisks indicate proteins containing all three conserved active site cysteine residues, all other Sulfobacillus sequences contain only the second and third residues. Protein tree adapted from SQR phylogeny laid out by Marcia et al. [77]. The tree is rooted midway to the sulfur oxygenase reductase from Thioalkalivibrio nitratireducens (YP 007217840), which was used as an outgroup.

Additional file 7: Figure S5. Phylogenetic analysis of tetrathionate hydrolase proteins (TTH). Sequences from Sulfobacillus genomes are listed in red. Diamonds indicate nodes with $>90 \%$ bootstrap support. Bootstrap values greater than $55 \%$ are shown as text. Protein tree adapted from Protze et al. [86].

Additional file 8: Figure S6. Phylogenetic analysis of DMSO-reductase superfamily of proteins. Sequences from Sulfobacillus genomes are listed in red. Diamonds indicate nodes with $>90 \%$ bootstrap support. Bootstrap values greater than $55 \%$ are shown as text. NapA/NasA, Periplasmic nitrate reductaes; Fdh, formate dehydrogenase; FdnG, formate-hydrogen lyase; NarG, dissimilatory nitrate reductases; EdbR/DdhA/SerA ethylbenzene dehydrogenase/dimethyl sulfide dehydrogenase/selenate reductase; DmsA, dimethyl-sulfoxide reductase; PsrA/PhsA, polysulfide/thiosulfate reductase; SreA, sulfur reductase, SoeA, sulfite-oxidase; TtrA, tetrathionate reductase.

Additional file 9: Figure S7. Phylogenetic analysis of hydrogenase superfamily of proteins. Sequences from Sulfobacillus genomes are listed in red. Diamonds indicate nodes with $>90 \%$ bootstrap support. Bootstrap values greater than $55 \%$ are shown as text.

Additional file 10: Figure S8. Phylogenetic analysis of RuBisCO proteins. Sequences from Sulfobacillus genomes are listed in red. Diamonds indicate nodes with $>90 \%$ bootstrap support. Bootstrap values greater than $55 \%$ are shown as text.

\section{Competing interests}

The authors declare that they have no competing interests.

\section{Authors' contributions}

$N B J, A N, C B, A S, B T$, and JFB analyzed the data. NBJ, AN, and JFB wrote and edited the manuscript. All authors read and approved the final manuscript.

\section{Acknowledgements}

This study was supported by the Genome Sciences Program in Carbon Cycling (contract DE-SC0004665), and Knowledgebase (DE-AC02-05CH11231) programs of the U.S. Department of Energy, Office of Science, Office of Biological and Environmental Research as well as the The Danish Council for Independent Research in Natural Sciences (FNU grant no. 11-107935). Sequence data of S. thermosulfidooxidans AT-1 (DSM 9293) were produced by the US Department of Energy Joint Genome Institute http://www.jgi.doe. gov/. We thank the late T. W. Arman (Iron Mountain Mines, Inc.) and R. Sugarek for access to the Richmond Mine and R. Carver and M. Jones for on-site assistance.

\section{Author details}

'Department of Earth and Planetary Science, University of California, Berkeley, CA 94720, USA. ${ }^{2}$ Present Address: Physical Biosciences Division, Lawrence Berkeley National Lab, Berkeley, CA, USA. ${ }^{3}$ Present Address: Section for Infection Microbiology, Department of Systems Biology, Technical University of Denmark, Lyngby, Denmark.

Received: 28 August 2014 Accepted: 27 November 2014 Published: 15 December 2014

\section{References}

1. Norris PR, Clark DA, Owen JP, Waterhouse S: Characteristics of Sulfobacillus acidophilus sp. nov. and other moderately thermophilic mineralsulphide-oxidizing bacteria. Microbiol-Sgm 1996, 142(Pt 4):775-783.

2. Li B, Chen Y, Liu Q, Hu S, Chen X: Complete genome analysis of Sulfobacillus acidophilus strain TPY, isolated from a hydrothermal vent in the Pacific Ocean. J Bacteriol 2011, 193:5555-5556.

3. Johnson DB, Okibe N, Roberto FF: Novel thermo-acidophilic bacteria isolated from geothermal sites in Yellowstone National Park: physiological and phylogenetic characteristics. Arch Microbiol 2003, 180:60-68.

4. Baker BJ, Banfield JF: Microbial communities in acid mine drainage. FEMS Microbiol Ecol 2003, 44:139-152.

5. Watling HR, Perrot FA, Shiers DW: Comparison of selected characteristics of Sulfobacillus species and review of their occurrence in acidic and bioleaching environments. Hydrometallurgy 2008, 93:57-65.

6. Bond PL, Druschel GK, Banfield JF: Comparison of acid mine drainage microbial communities in physically and geochemically distinct ecosystems. Appl Environ Microbiol 2000, 66:4962-4971.

7. Justice NB, Pan C, Mueller R, Spaulding SE, Shah V, Sun CL, Yelton AP, Miller CS, Thomas BC, Shah M, VerBerkmoes N, Hettich R, Banfield JF: Heterotrophic archaea contribute to carbon cycling in low-pH, suboxic biofilm communities. Appl Environ Microbiol 2012, 78:8321-8330.

8. Druschel GK, Baker BJ, Gihring TM, Banfield JF: Acid mine drainage biogeochemistry at Iron Mountain, California. Geochem Trans 2004, 5:13-32. 
9. Dick GJ, Andersson AF, Baker BJ, Simmons SL, Thomas BC, Yelton AP, Banfield JF: Community-wide analysis of microbial genome sequence signatures. Genome Biol 2009, 10:R85.

10. Melamud VS, Pivovarova TA, Tourova TP, Kolganova TV, Osipov GA, Lysenko AM, Kondrat'eva TF, Karavaiko Gl: Sulfobacillus sibiricus sp. nov., a New Moderately Thermophilic Bacterium - Springer. Microbiology 2003, 72:605-612.

11. Bogdanova TI, Tsaphna IA, Kondrat'eva TF, Duda VI, Suzina NE, Melamud VS, Tourova TP, Karavaiko Gl: Sulfobacillus thermotolerans sp. nov., a thermotolerant, chemolithotrophic bacterium. Int J Syst Evol Microbiol 2006, 56:1039-1042.

12. Golovacheva RS, Karavaiko Gl: Sulfobacillus, a new genus of thermophilic sporulating bacteria. Mikrobiologiia 1978, 47:815-822.

13. Johnson DB, Joulian C, d'Hugues P, Hallberg KB: Sulfobacillus benefaciens sp. nov., an acidophilic facultative anaerobic Firmicute isolated from mineral bioleaching operations. Extremophiles 2008, 12:789-798.

14. Bridge $T$, Johnson DB: Reduction of soluble iron and reductive dissolution of ferric iron-containing minerals by moderately thermophilic iron-oxidizing bacteria. Appl Environ Microbiol 1998, 64:2181-2186.

15. Tsaplina IA, Zhuravleva AE, Egorova MA, Bogdanova TI, Krasil'nikova EN, Zakharchuk LM, Kondrat'eva TF: Response to oxygen limitation in bacteria of the genus sulfobacillus. Microbiology 2010, 79:13-22.

16. Travisany D, Di Genova A, Sepulveda A, Bobadilla-Fazzini RA, Parada P, Maass A: Draft genome sequence of the Sulfobacillus thermosulfidooxidans Cutipay strain, an indigenous bacterium isolated from a naturally extreme mining environment in Northern Chile. J Bacteriol 2012, 194:6327-6328.

17. Anderson I, Chertkov O, Chen A, Saunders E, Lapidus A, Nolan M, Lucas S, Hammon N, Deshpande S, Cheng J-F, Han C, Tapia R, Goodwin LA, Pitluck S, Liolios K, Pagani I, Ivanova N, Mikhailova N, Pati A, Palaniappan K, Land M, Pan C, Rohde M, Pukall R, Göker M, Detter JC, Woyke T, Bristow J, Eisen JA, Markowitz $\vee$ et al: Complete genome sequence of the moderately thermophilic mineral-sulfide-oxidizing firmicute Sulfobacillus acidophilus type strain (NAL(T)). Stand Genomic Sci 2012, 6:1-13.

18. Guo X, Yin H, Liang Y, Hu Q, Zhou X, Xiao Y, Ma L, Zhang X, Qiu G, Liu X: Comparative genome analysis reveals metabolic versatility and environmental adaptations of Sulfobacillus thermosulfidooxidans strain ST. PLoS One 2014, 9:e99417.

19. Markowitz VM, Chen I-MA, Palaniappan K, Chu K, Szeto E, Pillay M, Ratner A, Huang J, Woyke T, Huntemann M, Anderson I, Billis K, Varghese N, Mavromatis K, Pati A, Ivanova NN, Kyrpides NC: IMG 4 version of the integrated microbial genomes comparative analysis system. Nucleic Acids Res 2014, 42(Database issue):D560-D567.

20. Denef VJ, Denef VJ, Kalnejais LH, Kalnejais LH, Mueller RS, Mueller RS, Wilmes P, Wilmes P, Baker BJ, Baker BJ, Thomas BC, Thomas BC, VerBerkmoes NC, VerBerkmoes NC, Hettich RL, Hettich RL, Banfield JF, Banfield JF: Proteogenomic basis for ecological divergence of closely related bacteria in natural acidophilic microbial communities. Proc Natl Acad Sci 2010, 107:2383-2390.

21. Miller CS, Baker BJ, Thomas BC, Singer SW, Banfield JF: EMIRGE: reconstruction of full-length ribosomal genes from microbial community short read sequencing data. Genome Biol 2011, 12:R44.

22. JGl: Protocols in Production Sequencing. [http://jgi.doe.gov/collaboratewith-jgi/pmooverview/protocols-sample-preparation-information/]

23. St John J: SeqPrep. [https://github.com/jstjohn/SeqPrep]

24. Denef VJ, Banfield JF: In situ evolutionary rate measurements show ecological success of recently emerged bacterial hybrids. Science 2012, 336:462-466.

25. Peng Y, Leung HCM, Yiu SM, Chin FYL: IDBA-UD: a de novo assembler for single-cell and metagenomic sequencing data with highly uneven depth. Bioinformatics 2012, 28:1420-1428.

26. Li H, Durbin R: Fast and accurate short read alignment with Burrows-Wheeler transform. Bioinformatics 2009, 25:1754-1760

27. Hyatt D, Chen G-L, LoCascio PF, Land ML, Larimer FW, Hauser L: Prodigal: prokaryotic gene recognition and translation initiation site identification. BMC Bioinformatics 2010, 11:119.

28. Lowe TM, Eddy SR: tRNAscan-SE: a program for improved detection of transfer RNA genes in genomic sequence. Nucleic Acids Res 1997, 25:955-964.

29. Altschul SF, Gish W, Miller W, Myers EW, Lipman DJ: Basic local alignment search tool. J Mol Biol 1990, 215:403-410.

30. Suzek BE, Huang H, McGarvey P, Mazumder R, Wu CH: UniRef: comprehensive and non-redundant UniProt reference clusters. Bioinformatics 2007, 23:1282-1288.
31. Ogata H, Goto S, Sato K, Fujibuchi W, Bono H, Kanehisa M: KEGG: Kyoto Encyclopedia of Genes and Genomes. Nucleic Acids Res 1999, 27:29-34

32. Quevillon E, Silventoinen V, Pillai S, Harte N, Mulder N, Apweiler R, Lopez R: InterProScan: protein domains identifier. Nucleic Acids Res 2005, 33:W116-W120.

33. Markowitz VM, Chen IMA, Palaniappan K, Chu K, Szeto E, Grechkin Y, Ratner A, Jacob B, Huang J, Williams P, Huntemann M, Anderson I, Mavromatis K, Ivanova NN, Kyrpides NC: IMG: the integrated microbial genomes database and comparative analysis system. Nucleic Acids Res 2011, 40:D115-D122.

34. Edgar RC: Search and clustering orders of magnitude faster than BLAST. Bioinformatics 2010, 26:2460-2461.

35. Edgar RC: MUSCLE: multiple sequence alignment with high accuracy and high throughput. Nucleic Acids Res 2004, 32:1792-1797.

36. Castresana J: Selection of conserved blocks from multiple alignments for their use in phylogenetic analysis. Mol Biol Evol 2000, 17:540-552.

37. Talavera G, Castresana J: Improvement of phylogenies after removing divergent and ambiguously aligned blocks from protein sequence alignments. Syst Biol 2007, 56:564-577.

38. Abascal F, Zardoya R, Posada D: ProtTest: selection of best-fit models of protein evolution. Bioinformatics 2005, 21:2104-2105.

39. Stamatakis A: RAxML-VI-HPC: Maximum likelihood-based phylogenetic analyses with thousands of taxa and mixed models. Bioinformatics 2006, 22:2688-2690.

40. Letunic I, Bork P: Interactive Tree Of Life (iTOL): an online tool for phylogenetic tree display and annotation. Bioinformatics 2007, 23:127-128

41. Sorek R, Zhu Y, Creevey CJ, Francino MP, Bork P, Rubin EM: Genome-wide experimental determination of barriers to horizontal gene transfer. Science 2007, 318:1449-1452.

42. Wu M, Eisen JA: A simple, fast, and accurate method of phylogenomic inference. Genome Biol 2008, 9:R151.

43. Pruesse E, Quast C, Knittel K, Fuchs BM, Ludwig W, Peplies J, Glockner FO: SILVA: a comprehensive online resource for quality checked and aligned ribosomal RNA sequence data compatible with ARB. Nucleic Acids Res 2007, 35:7188-7196.

44. Wright ES, Yilmaz LS, Noguera DR: DECIPHER, a search-based approach to Chimera identification for 16S rRNA sequences. Appl Environ Microbiol 2012, 78:717-725.

45. Edgar RC, Haas BJ, Clemente JC, Quince C, Knight R: UCHIME improves sensitivity and speed of chimera detection. Bioinformatics 2011, 27:2194-2200.

46. DeSantis TZ, Hugenholtz P, Larsen N, Rojas M, Brodie EL, Keller K, Huber T, Dalevi D, Hu P, Andersen GL: Greengenes, a chimera-checked 16S rRNA gene database and workbench compatible with ARB. Appl Environ Microbiol 2006, 72:5069-5072

47. Caporaso JG, Kuczynski J, Stombaugh J, Bittinger K, Bushman FD, Costello EK, Fierer N, Peña AG, Goodrich JK, Gordon Jl, Huttley GA, Kelley ST, Knights D, Koenig JE, Ley RE, Lozupone CA, McDonald D, Muegge BD, Pirrung M, Reeder J, Sevinsky JR, Turnbaugh PJ, Walters WA, Widmann J, Yatsunenko T, Zaneveld J, Knight R: QIIME allows analysis of high-throughput community sequencing data. Nat Methods 2010, 7:335-336.

48. Caporaso JG, Bittinger K, Bushman FD, DeSantis TZ, Andersen GL, Knight R: PyNAST: a flexible tool for aligning sequences to a template alignment. Bioinformatics 2010, 26:266-267.

49. Bond PL, Banfield JF: Design and performance of rRNA targeted oligonucleotide probes for in situ detection and phylogenetic identification of microorganisms inhabiting acid mine drainage environments. Microb Ecol 2001, 41:149-161.

50. Amann R, Ludwig W, Schleifer KH: Phylogenetic identification and in-situ detection of individual microbial-cells without cultivation. Microbio/ Rev 1995, 59:143-169.

51. Saraste M: Structure and evolution of cytochrome oxidase. Antonie Van Leeuwenhoek 1994, 65:285-287.

52. Beinert $\mathrm{H}$ : Copper $\mathrm{A}$ of cytochrome c oxidase, a novel, long-embattled, biological electron-transfer site. Eur J Biochem 1997, 245:521-532.

53. Borisov VB, Gennis RB, Hemp J, Verkhovsky Ml: The cytochrome bd respiratory oxygen reductases. BBA - Bioenergetics 1807, 2011:1398-1413.

54. Moparthi VK, Hägerhäll C: The evolution of respiratory chain complex I from a smaller last common ancestor consisting of 11 protein subunits. J Mol Evol 2011, 72:484-497. 
55. Kerscher S, Dröse S, Zickermann V, Brandt U: The three families of respiratory NADH dehydrogenases. Results Probl Cell Differ 2008, 45:185-222

56. Lancaster CRD: Succinate:quinone oxidoreductases: an overview. Biochim Biophys Acta 2002, 1553:1-6.

57. Schafer G, Anemuller S, Moll R: Archaeal complex II: 'classical' and "non-classical" succinate : quinone reductases with unusual features. BBA - Bioenergetics 2002, 1553:57-73.

58. Schippers A, Jozsa P, Sand W: Sulfur chemistry in bacterial leaching of pyrite. Appl Environ Microbiol 1996, 62:3424-3431.

59. Dopson M, Johnson DB: Biodiversity, metabolism and applications of acidophilic sulfur-metabolizing microorganisms. Environ Microbiol 2012, 14:2620-2631

60. Luther GW: Pyrite oxidation and reduction: molecular orbital theory considerations. Geochim Cosmochim Acta 1987, 51:3193-3199.

61. Urich T, Gomes CM, Kletzin A, Frazão C: X-ray structure of a self-compartmentalizing sulfur cycle metalloenzyme. Science 2006 311:996-1000.

62. Chen L, Ren Y, Lin J, Liu X, Pang X, Lin J: Acidithiobacillus caldus sulfur oxidation model based on transcriptome analysis between the wild type and sulfur oxygenase reductase defective mutant. PLoS One 2012 7:e39470.

63. Zhang $H$, Guo W, Xu C, Zhou H, Chen X: Site-specific mutagenesis and functional analysis of active sites of sulfur oxygenase reductase from Gram-positive moderate thermophile Sulfobacillus acidophilus TPY. Microbiol Res 2013, 168:654-660.

64. Hedderich R, Forzi L: Energy-converting [NiFe] hydrogenases: more than just $\mathrm{H}_{2}$ activation. J Mol Microbiol Biotechnol 2005, 10:92-104.

65. Grein F, Ramos AR, Venceslau SS, Pereira IAC: Unifying concepts in anaerobic respiration: insights from dissimilatory sulfur metabolism. Biochim Biophys Acta 1827, 2013:145-160.

66. Hedderich R, Klimmek O, Kröger A, Dirmeier R, Keller M, Stetter KO: Anaerobic respiration with elemental sulfur and with disulfides. FEMS Microbiol Rev 1999, 22:353-381.

67. Quatrini R, Appia-Ayme C, Denis Y, Jedlicki E, Holmes DS, Bonnefoy V: Extending the models for iron and sulfur oxidation in the extreme Acidophile Acidithiobacillus ferrooxidans. BMC Genomics 2009, 10:394.

68. Rohwerder T: The sulfane sulfur of persulfides is the actual substrate of the sulfur-oxidizing enzymes from Acidithiobacillus and Acidiphilium spp. Microbiology 2003, 149:1699-1710.

69. Rohwerder T, Sand W: Oxidation of inorganic sulfur compounds in acidophilic prokaryotes. Eng Life Sci 2007, 7:301-309.

70. Hamann N, Mander GJ, Shokes JE, Scott RA, Bennati M, Hedderich R: A cysteine-rich CCG domain contains a novel [4Fe-4S] cluster binding motif as deduced from studies with subunit B of heterodisulfide reductase from Methanothermobacter marburgensis. Biochemistry 2007, 46:12875-12885

71. Stockdreher $Y$, Sturm M, Josten M, Sahl H-G, Dobler N, Zigann R, Dahl C: New proteins involved in sulfur trafficking in the cytoplasm of allochromatium vinosum. J Biol Chem 2014, 289:12390-12403.

72. Stockdreher $Y$, Venceslau SS, Josten M, Sahl H-G, Pereira IAC, Dahl C: Cytoplasmic sulfurtransferases in the purple sulfur bacterium allochromatium vinosum: evidence for sulfur transfer from DsrEFH to DsrC. PLoS One 2012, 7:e40785.

73. Morris TW, Reed KE, Cronan JE: Identification of the gene encoding lipoate-protein ligase-a of escherichia-coli - molecular-cloning and characterization of the Lpla gene and gene-product. J Biol Chem 1994, 269:16091-16100.

74. Perham RN: Swinging arms and swinging domains in multifunctional enzymes: catalytic machines for multistep reactions. Annu Rev Biochem 2000, 69:961-1004

75. Liu L-J, Stockdreher Y, Koch T, Sun S-T, Fan Z, Josten M, Sahl H-G, Wang Q, Luo Y-M, Liu S-J, Dahl C, Jiang C-Y: Thiosulfate transfer mediated by DsrE/ TusA homologs from acidothermophilic sulfur-oxidizing archaeon metallosphaera cuprina. J Biol Chem 2014, 289:26949-26959.

76. Strittmatter AW, Liesegang H, Rabus R, Decker I, Amann J, Andres S, Henne A, Fricke WF, Martinez-Arias R, Bartels D, Goesmann A, Krause L, Puehler A, Klenk H-P, Richter M, Schueler M, Gloeckner FO, Meyerdierks A, Gottschalk $G$, Amann R: Genome sequence of Desulfobacterium autotrophicum HRM2, a marine sulfate reducer oxidizing organic carbon completely to carbon dioxide. Environ Microbiol 2009, 11:1038-1055.
77. Marcia M, Ermler U, Peng G, Michel H: A new structure-based classification of sulfide:quinone oxidoreductases. Proteins: Struct Funct Bioinformatics 2009, 78:1073-1083.

78. Wakai S, Tsujita M, Kikumoto M, Manchur MA, Kanao T, Kamimura K Purification and characterization of sulfide: quinone oxidoreductase from an acidophilic iron-oxidizing bacterium, Acidithiobacillus ferrooxidans. Biosci Biotechnol Biochem 2007, 71:2735-2742.

79. Cherney MM, Zhang Y, Solomonson M, Weiner JH, James MNG: Crysta structure of sulfide: quinone oxidoreductase from acidithiobacillus ferrooxidans: insights into sulfidotrophic respiration and detoxification. J Mol Biol 2010, 398:292-305.

80. Lencina AM, Ding Z, Schurig-Briccio LA, Gennis RB: Characterization of the type III sulfide:quinone oxidoreductase from Caldivirga maquilingensis and its membrane binding. Biochim Biophys Acta 1827, 2013:266-275.

81. De Jong GA, Hazeu W, Bos P, Kuenen JG: Isolation of the tetrathionate hydrolase from Thiobacillus acidophilus. Eur J Biochem 1997, 243:678-683.

82. Kanao T, Kamimura K, Sugio T: Identification of a gene encoding a tetrathionate hydrolase in Acidithiobacillus ferrooxidans. J Biotechnol 2007, 132:16-22.

83. Bugaytsova Z, Lindström EB: Localization, purification and properties of a tetrathionate hydrolase from Acidithiobacillus caldus. Eur J Biochem 2004, 271:272-280.

84. Egorova MA, Tsaplina IA, Zakharchuk LM, Bogdanova TI, Krasil'nikova EN: Effect of cultivation conditions on the growth and activities of sulfur metabolism enzymes and carboxylases of Sulfobacillus thermosulfidooxidans subsp asporogenes strain 41. Appl Biochem Microbiol 2004, 40:381-387.

85. Shiers DW, Ralph DE, Watling HR: A comparative study of substrate utilisation by Sulfobacillus species in mixed ferrous ion and tetrathionate growth medium. Hydrometallurgy 2010, 104:363-369.

86. Protze J, Müller F, Lauber K, Naß B, Mentele R, Lottspeich F, Kletzin A: An extracellular tetrathionate hydrolase from the thermoacidophilic archaeon acidianus ambivalens with an activity optimum at $\mathrm{pH} 1$. Front Microbiol 2011, 2:68

87. Müller FH, Bandeiras TM, Urich T, Teixeira M, Gomes CM, Kletzin A: Coupling of the pathway of sulphur oxidation to dioxygen reduction: characterization of a novel membrane-bound thiosulphate:quinone oxidoreductase. Mol Microbiol 2004, 53:1147-1160.

88. Rothery RA, Workun GJ, Weiner JH: The prokaryotic complex iron-sulfur molybdoenzyme family. Biochim Biophys Acta 2008, 1778:1897-1929.

89. Hinsley AP, Berks BC: Specificity of respiratory pathways involved in the reduction of sulfur compounds by Salmonella enterica. Microbiol-Sgm 2002, 148:3631-3638

90. Guiral M, Tron P, Aubert C, Gloter A, lobbi-Nivol C, Giudici-Orticoni M-T: A membrane-bound multienzyme, hydrogen-oxidizing, and sulfur-reducing complex from the hyperthermophilic bacterium Aquifex aeolicus. J Biol Chem 2005, 280:42004-42015.

91. Dahl C, Franz B, Hensen D, Kesselheim A, Zigann R: Sulfite oxidation in the purple sulfur bacterium Allochromatium vinosum: identification of SoeABC as a major player and relevance of SoxYZ in the process. Microbiol-Sgm 2013, 159:2626-2638.

92. Kopriva S, Buchert T, Fritz G, Suter M, Benda RD, Schunemann V, Koprivova $A$, Schurmann $P$, Trautwein AX, Kroneck $P$, Brunold $C$ : The presence of an iron-sulfur cluster in adenosine 5 '-phosphosulfate reductase separates organisms utilizing adenosine 5 '-phosphosulfate and phosphoadenosine 5'-phosphosulfate for sulfate assimilation. J Biol Chem 2002, 277:21786-21791.

93. Bick JA, Dennis JJ, Zylstra GJ, Nowack J, Leustek T: Identification of a new class of 5 -adenylylsulfate (APS) reductases from sulfate-assimilating bacteria. J Bacteriol 2000, 182:135-142.

94. Bonnefoy $V$, Holmes DS: Genomic insights into microbial iron oxidation and iron uptake strategies in extremely acidic environments. Environ Microbiol 2011, 14:1597-1611.

95. Coupland $\mathrm{K}$, Johnson DB: Evidence that the potential for dissimilatory ferric iron reduction is widespread among acidophilic heterotrophic bacteria. FEMS Microbiol Lett 2008, 279:30-35.

96. Johnson DB, Kanao T, Hedrich S: Redox transformations of iron at extremely low pH: fundamental and applied aspects. Front Microbiol 2012, 3:1-13.

97. Ohmura N, Sasaki K, Matsumoto N, Saiki H: Anaerobic respiration using Fe3 ,$+ \mathrm{SO}$, and $\mathrm{H} 2$ in the chemolithoautotrophic bacterium acidithiobacillus ferrooxidans. J Bacteriol 2002, 184:2081-2087. 
98. Kucera J, Bouchal P, Cerna H, Potesil D, Janiczek O, Zdrahal Z, Mandl M: Kinetics of anaerobic elemental sulfur oxidation by ferric iron in Acidithiobacillus ferrooxidans and protein identification by comparative 2-DE-MS/MS. Antonie Van Leeuwenhoek 2011, 101:561-573.

99. Osorio H, Mangold S, Denis Y, Nancucheo I, Esparza M, Johnson DB, Bonnefoy V, Dopson M, Holmes DS: Anaerobic sulfur metabolism coupled to dissimilatory iron reduction in the extremophile acidithiobacillus ferrooxidans. Appl Environ Microbiol 2013, 79:2172-2181.

100. Thamdrup B, Finster K, Hansen JW, Bak F: Bacterial disproportionation of elemental sulfur coupled to chemical reduction of iron or manganese. Appl Environ Microbiol 1993, 59:101-108.

101. Hardisty DS, Olyphant GA, Bell JB, Johnson AP, Pratt LM: Acidophilic sulfur disproportionation. Geochim Cosmochim Acta 2013, 113(C):136-151.

102. Karavaiko GI, Krasil'nikova EN, Tsaplina IA, Bogdanova TI, Zakharchuk LM: Growth and carbohydrate metabolism of sulfobacilli. Microbiology 2001, 70:245-250

103. Vignais PM, Billoud B: Occurrence, classification, and biological function of hydrogenases: an overview. Chem Rev 2007, 107:4206-4272.

104. Constant P, Chowdhury SP, Pratscher J, Conrad R: Streptomycetes contributing to atmospheric molecular hydrogen soil uptake are widespread and encode a putative high-affinity [NiFe]-hydrogenase. Environ Microbiol 2010, 12:821-829.

105. Constant P, Chowdhury SP, Hesse L, Pratscher J, Conrad R: Genome data mining and soil survey for the novel group $5 \mathrm{NiFe}$-hydrogenase to explore the diversity and ecological importance of presumptive high-affinity H-2-oxidizing bacteria. Appl Environ Microbiol 2011, 77:6027-6035.

106. Thauer RK, Kaster A-K, Goenrich M, Schick M, Hiromoto T, Shima S: Hydrogenases from methanogenic archaea, nickel, a novel cofactor, and H 2Storage. Annu Rev Biochem 2010, 79:507-536.

107. Coppi MV: The hydrogenases of Geobacter sulfurreducens: a comparative genomic perspective. Microbiol-Sgm 2005, 151:1239-1254.

108. Marreiros BC, Batista AP, Duarte AMS, Pereira MM: A missing link between complex I and group 4 membrane-bound [NiFe] hydrogenases. BBA - Bioenergetics 1827, 2013:198-209.

109. King GM, Weber CF: Distribution, diversity and ecology of aerobic CO-oxidizing bacteria. Nat Rev Microbiol 2007, 5:107-118.

110. Lin JT, Lin JT, Stewart V, Stewart V: Nitrate assimilation by bacteria. Adv Microb Physiol 1998, 39:1-30. 379.

111. Richardson DJ, Berks BC, Russell DA, Spiro S, Taylor CJ: Functional, biochemical and genetic diversity of prokaryotic nitrate reductases. Cell Mol Life Sci 2001, 58:165-178.

112. Gardner PR: Nitric oxide dioxygenase function and mechanism of flavohemoglobin, hemoglobin, myoglobin and their associated reductases. J Inorg Biochem 2005, 99:247-266.

113. Poole RK, Hughes MN: New functions for the ancient globin family: bacterial responses to nitric oxide and nitrosative stress. Mol Microbiol 2000, 36:775-783

114. Caldwell PE, MacLean MR, Norris PR: Ribulose bisphosphate carboxylase activity and a Calvin cycle gene cluster in Sulfobacillus species. Microbiol-Sgm 2007, 153:2231-2240.

115. Tabita FR, Hanson TE, Li HY, Satagopan S, Singh J, Chan S: Function, structure, and evolution of the RubisCO-like proteins and their RubisCO homologs. Microbiol Mol Biol Rev 2007, 71:576-599.

116. Barrie Johnson D, Hallberg KB: Carbon, Iron and Sulfur Metabolism in Acidophilic Micro-Organisms. In Advances in Microbial Physiology, volume 54. Edited by Poole RK. Elsevier; 2008:201-255 [Advances in Microbial Physiology]

117. Sugiyama M, Suzuki S-I, Tonouchi N, Yokozeki K: Transaldolase/glucose-6phosphate isomerase bifunctional enzyme and ribulokinase as factors to increase xylitol production from D-arabitol in Gluconobacter oxydans. Biosci Biotechnol Biochem 2003, 67:2524-2532.

118. Kim S, Lee SB: Identification and characterization of the bacterial d-gluconate dehydratase in Achromobacter xylosoxidans. Biotechnol Bioprocess Eng 2008, 13:436-444.

119. Krasil'nikova EN, Zakharchuk LM, Egorova MA, Bogdanova TI, Zhuravleva AE, Tsaplina IA: Regulation of metabolic pathways in sulfobacilli under different aeration regimes. Microbiology 2010, 79:147-152.

120. Nancucheo I, Johnson DB: Production of glycolic acid by chemolithotrophic iron- and sulfur-oxidizing bacteria and its role in delineating and sustaining acidophilic sulfide mineral-oxidizing consortia. Appl Environ Microbiol 2010, 76:461-467.
121. Chai $Y$, Kolter $R$, Losick $R$ : A widely conserved gene cluster required for lactate utilization in bacillus subtilis and its involvement in biofilm formation. J Bacteriol 2009, 191:2423-2430.

122. Tholozan JL, Touzel JP, Samain E, Grivet JP, Prensier G, Albagnac G: Clostridium neopropionicum sp. nov., a strict anaerobic bacterium fermenting ethanol to propionate through acrylate pathway. Arch Microbiol 1992, 157:249-257.

123. Schink B, Kremer DR, Hansen TA: Pathway of propionate formation from ethanol in Pelobacter propionicus. Arch Microbiol 1987, 147:321-327.

124. Tsaplina IA, Osipov GA, Bogdanova TI, NEDOREZOVA TP, Karavaiko Gl: Fatty-acid composition of lipids in thermoacidophilic bacteria of the genus sulfobacillus. Microbiology 1994, 63:459-464.

125. Kaneda T: Iso-and anteiso-fatty acids in bacteria: biosynthesis, function, and taxonomic significance. Microbiol Rev 1991, 55:288-302.

126. Oshima M, Ariga T: Omega-cyclohexyl fatty-acids in acidophilic thermophilic bacteria - studies on their presence, structure, and biosynthesis using precursors labeled with stable isotopes and radioisotopes. J Biol Chem 1975, 250:6963-6968.

127. Duda VI, Suzina NE, Severina LO, Dmitriev W, Karavaiko Gl: Formation of flat lamellar intramembrane lipid structures in microorganisms. J Membr Biol 2001, 180:33-48.

128. Kannenberg EL, Poralla K: Hopanoid biosynthesis and function in bacteria. Naturwissenschaften 1999, 86:168-176.

129. Spanova M, Daum G: Squalene - biochemistry, molecular biology, process biotechnology, and applications. Eur J Lipid Sci Technol 2011, 113:1299-1320.

130. Jones DS, Jones DS, Albrecht HL, Albrecht HL, Dawson KS, Dawson KS, Schaperdoth I, Schaperdoth I, Freeman KH, Freeman KH, Pi Y, Pi Y, Pearson A, Pearson A, Macalady JL, Macalady JL: Community genomic analysis of an extremely acidophilic sulfur-oxidizing biofilm. ISME J 2011, 6:158-170.

131. Welander PV, Doughty DM, Wu C-H, Mehay S, Summons RE, Newman DK: Identification and characterization of Rhodopseudomonas palustris TIE-1 hopanoid biosynthesis mutants. Geobiology 2012, 10:163-177.

132. Cárdenas JP, Moya F, Covarrubias P, Shmaryahu A, Levicán G, Holmes DS, Quatrini R: Comparative genomics of the oxidative stress response in bioleaching microorganisms. Hydrometallurgy 2012, 127-128(C):162-167.

133. Norambuena J, Flores R, Cárdenas JP, Quatrini R, Chávez R, Levicán G: Thiol/ disulfide system plays a crucial role in redox protection in the acidophilic iron-oxidizing bacterium leptospirillum ferriphilum. PLoS One 2012, 7:e44576.

134. Empadinhas N, da Costa MS: Diversity and biosynthesis of compatible solutes in hyper/thermophiles. Int Microbio/ 2006, 9:199-206.

135. Mosier AC, Justice NB, Bowen BP, Baran R, Thomas BC, Northen TR, Banfield JF: Metabolites associated with adaptation of microorganisms to an acidophilic, metal-rich environment identified by stable-isotope-enabled metabolomics. MBio 2013, 4:e00484-12

136. Bren A, Eisenbach M: How signals are heard during bacterial chemotaxis: protein-protein interactions in sensory signal propagation. $J$ Bacteriol 2000, 182:6865-6873.

137. Ghauri MA, Johnson DB: Physiological diversity amongst some moderately thermophilic iron-oxidizing bacteria. FEMS Microbiol Ecol 1991, 85:327-334

138. Wood AP, Kelly DP: Autotrophic and mixotrophic growth of three thermoacidophilic iron-oxidizing bacteria. FEMS Microbiol Lett 1983, 20:107-112.

139. Wood AP, Kelly DP: Growth and sugar metabolism of a thermoaddophilic iron-oxidizing mixotrophic bacterium. Microbiology 1984, 130:1337-1349.

140. Tsaplina IA, Krasil'nikova EN, Zhuravleva AE, Egorova MA, Zakharchuk LM Suzina NE, Duda VI, Bogdanova TI, Stadnichuk IN, Kondrat'eva TF: The dependence of intracellular ATP level on the nutrition mode of the acidophilic bacteria Sulfobacillus thermotolerans and Alicyclobacillus tolerans. Microbiology 2008, 77:654-664.

141. Ma S, Banfield JF: Micron-scale Fe2+/Fe3+, intermediate sulfur species and $\mathrm{O} 2$ gradients across the biofilm-solution-sediment interface control biofilm organization. Geochim Cosmochim Acta 2011, 75:3568-3580.

142. Druschel GK, Hamers RJ, Banfield JF: Kinetics and mechanism of polythionate oxidation to sulfate at low pH by $\mathrm{O} 2$ and Fe3. Geochim Cosmochim Acta 2003, 67:4457-4469.

\section{doi:10.1186/1471-2164-15-1107}

Cite this article as: Justice et al.: Comparison of environmental and isolate Sulfobacillus genomes reveals diverse carbon, sulfur, nitrogen, and hydrogen metabolisms. BMC Genomics 2014 15:1107. 